\title{
Renormalization Group Flow of Two-Dimensional Hierarchical Heisenberg Model Of Dyson-Wilson Type
}

\author{
Keiichi R. Ito \\ Department of Mathematics, College of Liberal Arts, Kyoto University, Kyoto 606, Japan \\ and Konan College of Women, Takaya-Chou, Konan City, Aichi Pref. 483, Japan ${ }^{\star \star}$
}

Received January 25, 1990; in revised form July 20, 1990

\begin{abstract}
Two-dimensional $O(N)$-invariant hierarchical Heisenberg models (of Dyson-Wilson type) are investigated by the real space renormalization group method. It is established that if $N \geqq 2$, then the effective actions at long distance scales are driven into the high temperature region by the iterative use of the block spin transformations, thus concluding the non-existence of phase transitions in the system. The correlation functions are also obtained and they decay at the speed of massive gaussian field model. The driving force is geometrical and an a priori one and stronger than the boundary effect which is bounded by $O(1)$ in the present system. Thus the hierarchical formulas fail to exhibit the Kosterlitz-Thouless transitions (at least for $N=2$ ).
\end{abstract}

\section{Introduction}

Conjectures of quark confinment in four-dimensional (4D) nonabelian lattice gauge theories and mass generations in 2D Heisenberg models are long standing problems in modern physics [1-3]. These problems may be solved only by hard analysis like real space renormalization group methods [4-7]. However this is difficult in these problems because these phenomena are non-perturbative. Thus we need to find a reason why the renormalization group flows are attracted into the high-temperature region (if our conjectures are true), and to develop a method which enables us to follow the trajectories in a non-perturbative way.

Our main result in this paper is that the renormalization group flow of two-dimensional $O(N)$-symmetric Heisenberg model is driven into the hightemperature region, within the hierarchical approximation of Dyson-Wilson type, without exhibiting the Kosterlitz-Thouless transitions for any initial $g_{0}(x)$. This

\footnotetext{
* Postal address

E-mail: ito@kurims.kyoto-u.ac.jp

$\star \star$ Permanent address
} 
result holds not only for $N \geqq 3$ but also for $N=2$. Then the formula fails to exhibit the KT transitions. We show that the driving force is geometrical and an a priori one and is stronger in the boundary effects in the hierarchical approximation. This model was first considered by Gawedzki and Kupiainen [6] and they gave an explicit solution for $N=\infty$. See also [7-9] for earlier reports on this subject.

In Sect. 2, we first propose a 2D $N$-vector model (Heisenberg model with $N$ components) defined on a hierarchical lattice embedded in two-dimensional lattice space $Z^{2}$. The hierarchical lattices constructed in this paper are called DysonWilson type and believed to be close to the real system [4-7]. Essential differences are that long-distance interactions are introduced to make the hierarchical structures and that boundary effects are bounded by $O(1)$ through all the distance scales and the magnitudes of the block spin variables.

In Sect. 3 we show that if $N \geqq 3$, the effective actions obey some a priori bounds which are essentially asymptotic freedom and have a probabilistic origin: balls hardly take the same directions compared with sticks and disks. However we can prove that the $O(2)$-model equally satisfies the same type of the bound, by considering larger blocks. Then it is difficult for the recursion formulas to find the difference.

We first consider $O(N)$ models, $N \geqq 4$, in Sects. 4 and 5 , and analyze the renormalization-group flows by the standard block-spin transformations of Wilson-Kadanoff type. The flow consists of three parts, depending on the number of renormalization group iterations and the typical magnitude of the field variables. For small numbers of the iterations, the flow is slowly attracted into the high temperature region in the large field region [4-5].

Once the flow is attracted into the high temperature region, then the flow approaches the origin exponentialy in $n$. This is consistent with the well known large deviation theory $[11,12]$ in probability theory. There is also a transition region between these two regions.

In Sect. 6 we investigate the correlation functions and show that $\langle\vec{\phi}(x) \vec{\phi}(y)\rangle \sim$ const $[d(x, y)]^{-2}$, no matter how low the initial temperature is (const may depend on it), where $d(x, y)$ is the hierarchical distance. The inverse-square decay is due to the built-in long-range interactions, and is consistent with the conjectured exponential clusterings in the Heisenberg model [12]. In Sect. 7, we discuss $N=2$ and 3 models in which we have weaker bounds. But we can prove the absence of the KT phase by the same method.

In Sect. 8, we argue the possibility of the application of the present method of analysis to the real systems, and in the appendix we prove some technical lemmas used in the paper.

\section{Construction of Model(s)}

The Gibbs measure of $N$-vector model in a finite volume $\Lambda\left(=\left[-L^{K} / 2, L^{K} / 2\right]^{2} \cap Z^{2}\right.$, where $L$ is an integer larger than 2 and $K$ is an arbitrarily large integer) is given by

$\langle\cdot\rangle=\frac{1}{Z(\Lambda)} \int \exp \left[-H_{\Lambda}(\{\vec{\phi}\})\right](\cdot) \prod_{x \in \Lambda} d \vec{\phi}(x)$,

where $\vec{\phi}(x)={ }^{t}\left(\phi_{1}(x), \ldots, \phi_{N}(x)\right) \in R^{N}$ is the random (spin) variable at the lattice point 
$x \in \Lambda$, and

$$
\begin{aligned}
H_{\Lambda} & =\sum_{\substack{(x, y) \in \Lambda \\
|x-y|=1}}(\vec{\phi}(x)-\vec{\phi}(x))^{2}+\sum_{x \in \Lambda} V_{0}\left(\vec{\phi}^{2}(x)\right) \\
& \equiv\left(\phi,\left(-\Delta_{\Lambda}\right) \phi\right)+\sum V_{0}\left(\phi^{2}(x)\right)
\end{aligned}
$$

is the finite volume Hamiltonian, $\sum_{(x, y)}$ stands for the pairwise sum $((x, y)$ is identified with $(y, x)), \Delta_{\Lambda}$ the lattice Laplacian operator and $V\left(\phi^{2}\right)$ is the self-interaction term. We assume that

$$
g_{0}\left(\varphi^{2}\right)=\exp \left[-V_{0}\left(\varphi^{2}\right)\right]
$$

decreases sufficiently rapidly (faster than any Gaussian functions of $\vec{\phi}$ ) as $\varphi^{2}=\vec{\phi}^{2} \rightarrow \infty$. Define the block spin operator $C$ by

$$
(C \vec{\phi})(x)=L^{-2} \sum_{y \in \square(L x)} \vec{\phi}(y)
$$

where $\square(L x)$ is the square of size $L \times L$, with the center at $L x \in L Z^{2} \cap \Lambda$. Thus the effective interaction at the distance scale $L^{n}$ is defined by

$$
\begin{aligned}
\mu_{n}(\vec{\psi}) & =\text { const } \times \exp \left[-H_{n}(\vec{\psi})\right]=\mathscr{R}\left(\mu_{n-1}\right)(\vec{\psi}) \\
& =\int \prod_{x \in \Lambda_{n}} \delta[\vec{\psi}(x)-(C \vec{\phi})(x)] \mu_{n-1}(\vec{\phi}) \prod_{x \in \Lambda_{n-1}} d \vec{\phi}(x),
\end{aligned}
$$

where $\mu_{0}(\psi)=$ const $\exp \left[-H_{0}\right], H_{0}=H_{\Lambda}$ and $\Lambda_{n}=L^{-n} \Lambda \cap Z^{2}$.

In repeating the transformations (2.6), one encounters the difficulty that $H_{n}(\vec{\phi})$ contains complicated non-local terms.

Thus we approximate the system by replacing the original Laplacian (2.2) by one of the following hierarchical ones:

model I (diagonalizable model):

$$
\begin{aligned}
& \left\langle\vec{\phi},\left(-\Delta_{h c L}\right) \vec{\phi}\right\rangle=H_{0}^{h c L} \\
& =\frac{1}{4 L^{2}} \sum_{n=1}^{K} \sum_{\substack{x \in \Lambda_{n} \\
n}} \sum_{\substack{\left.y, y^{\prime}\right) \in \square(L x) \\
y, y^{\prime} \in \Lambda_{n-1}}}\left[\left(C^{n-1} \vec{\phi}\right)(y)-\left(C^{n-1} \vec{\phi}\right)\left(y^{\prime}\right)\right]^{2} \\
& =\frac{1}{4 L^{2}}\left\{\sum_{x \in \Lambda_{1}} \sum_{\left(y, y^{\prime}\right) \in \square(L x)}\left(\vec{\phi}(y)-\vec{\phi}\left(y^{\prime}\right)\right)^{2}+\cdots\right\} \text {. }
\end{aligned}
$$

model II (non-diagonalizable model: general type):

$$
\langle\vec{\phi},(-\Delta) \vec{\phi}\rangle \rightarrow H_{0}^{h c L}=\sum_{n=1}^{K} \sum_{x \in \Lambda_{n}} H\left(\left(C^{n} \vec{\phi}\right)\left(y_{1}\right), \ldots,\left(C^{n} \vec{\phi}\right)\left(y_{r}\right)\right),
$$

where $y_{1}, \ldots, y_{r} \in \square(L x), r=L^{2}$ and the function $H$ satisfies

$$
\begin{aligned}
\left.H\left(G \vec{\phi}_{1}, \ldots, G \vec{\phi}_{r}\right)\right) & =H\left(\vec{\phi}_{1}, \ldots, \vec{\phi}_{r}\right), \text { for any } G \in O(N), \\
H\left(\vec{\phi}_{1}+\vec{\phi}_{1}, \ldots, \vec{\phi}_{r}+\vec{\phi}\right) & =H\left(\vec{\phi}_{1}, \ldots, \vec{\phi}_{r}\right), \\
\left|\frac{\partial H\left(\left\{x_{j k}\right\}\right)}{\partial x_{i i}}\right| & \leqq C_{1}\left(x_{i i}\right)^{-\delta}+C_{2},
\end{aligned}
$$


where $0<\delta<1$ and $C_{i}>0$. We also need the integrability though this is ensured by $\exp \left[-V_{0}\right]$. The property $(2.9 \mathrm{~b})$ is needed only to approximate the Laplacian and has no direct effects on the flow.

Our main conclusion does not depend on the choice of the hierarchical Hamiltonians. As is seen from the construction, $\Lambda_{n}(n=0,1, \ldots)$ is decomposed into $\left(L^{K-n}\right)^{2}$ squares of size $L \times L$, and the interaction at distance scale $L^{n}$ is restricted to these squares. To see that the hierarchical Laplacians approximate the real one, we consider

$$
\begin{aligned}
& \int \exp [i \vec{\alpha}(\vec{\phi}(x)-\vec{\phi}(y))] d v_{0}^{h c L} \\
& d v_{0}^{h c L}=(Z)^{-1} \exp \left[-H_{0}^{h c L}\right] \prod_{x \in \Lambda} d \vec{\phi}(x) .
\end{aligned}
$$

Here $x, y \in \Lambda$ and $Z$ is the normalization constant (introduce a mass term and remove it after taking the thermodynamic limit). Let

$$
d(x, y)=\min _{k \in N}\left\{L^{k} ; \text { both } L^{-k} x \text { and } L^{-k} y \in \square(m) \text { for some } m \in Z^{2}\right\} .
$$

$d(x, y)$ play the role of distance (hierarchical distance) and is the size of the smallest hierarchical square containing $x$ and $y$.

Assume $d(x, y)=L^{n}$, and consider the change of the variables:

$$
\left(C^{k} \vec{\phi}\right)(x)=\left(C^{k+1} \vec{\phi}\right)(\tilde{x})+\vec{z}(x) \text {, }
$$

where $\tilde{x} \in \Lambda_{k+1}$ is chosen so that $x \in \square(L \tilde{x})$, and thus $\left(C^{k+1} \phi\right)(\tilde{x})$ is independent of $x \in \square(L \tilde{x})$, and $z(x)$ are the fluctuation fields around $\left(C^{k+1} \phi\right)(\tilde{x})$ satisfying $(C z)(\tilde{x})=0$. If $k \geqq n$, then both $x$ and $y$ are contained in the same hierarchical square and thus $\left(C^{k} \phi\right)(\tilde{x})=\left(C^{k} \phi\right)(\tilde{y})$ and there exist no contributions from larger distance scale parts, see (2.7). Thus in the case of model I in which $d v_{0}^{h c L}$ is Gaussian, we find

$$
\text { Eq. }(2.10)=r^{(n-2)} r_{0}=\exp \left[-\operatorname{const}(\vec{\alpha})^{2}\left(\log _{L} d(x, y)+O(1)\right)\right]
$$

where const $>0$ and

$$
\begin{aligned}
& r=\left(Z^{\prime}\right)^{-1} \int \delta\left[\sum_{w \in \square(0)} \vec{z}(w)\right] \exp \left[i(\vec{\alpha}, \vec{z}(0))-\frac{1}{4 L^{2}} \sum_{\left(w, w^{\prime}\right)}\left(\vec{z}(w)-\vec{z}\left(w^{\prime}\right)\right)^{2}\right] D \vec{z}, \\
& r_{0}=\left(Z^{\prime}\right)^{-1} \int \delta\left[\sum_{w \in \square(0)} \vec{z}(w)\right] \exp \left[i(\vec{\alpha}, \vec{z}(i)-\vec{z}(j))-\frac{1}{4 L^{2}} \sum_{\left(w, w^{\prime}\right)}\left(\vec{z}(w)-\vec{z}\left(w^{\prime}\right)\right)^{2}\right] D \vec{z}
\end{aligned}
$$

with $D \vec{z}=\prod_{w \in \square(0)} d \vec{z}(w)$. Here we have used the facts that the fluctuation fields are not coupled if they belong to different hierarchical squares and that all points in the square are identical, and finally $i$ and $j$ in the second equation are relative positions of $x$ and $y$ in $\square(0) \subset \Lambda_{n}$. Then $d v_{0}^{h c L}$ for the model I approximates the original one with a reasonable accuracy. The measure $d v_{0}^{h c L}$ for model II can be similarly discussed and it will be easily seen that Eq. (2.10) behaves like $\exp [-$ const $\log d(x, y)]$.

Remarks 1. (1) Because of the hierarchical structure in $H_{0}^{\text {hcL }}$, there exists a long-range interaction: Add a mass term $\sum_{x} m^{2} \phi^{2}(x)$ to $H_{0}^{h c L}$, where $m^{2}>0$. Note that $\sum \phi^{2}(x)$ 
equals

$$
L^{2} \sum_{x \in \Lambda_{1}}(C \vec{\phi})^{2}(x)+2 \sum_{x \in \Lambda_{1}}(C \vec{\phi})(x)\left[\sum_{y \in \square(L x)} \vec{z}(y)\right]+\sum_{x \in \Lambda_{1}} \sum_{y \in \square(L x]} \vec{z}^{2}(y),
$$

where $\sum_{y \in \square(L x]} \vec{z}(y)=0$. Thus

$$
\langle\vec{\phi}(x) \vec{\phi}(y)\rangle=O\left(L^{-2 n}\right)=O\left(d^{-2}(x, y)\right) .
$$

(2) By replacing $\left[\left(C^{n} \phi\right)(y)-\left(C^{n} \phi\right)\left(y^{\prime}\right)\right]^{2}$ in Eq. (2.7) by

$$
L^{(d-2) n}\left[\left(\tilde{C}^{n} \phi\right)(y)-\left(\tilde{C}^{n} \phi\right)\left(y^{\prime}\right)\right]^{2}
$$

one has the $d$-dimensional model $[6,7]$, where $(\tilde{C} \phi)(x)=L^{-d} \sum \phi(L x+\zeta)$. Then $C=\widetilde{C}$ for $d=2$. Set $L=2^{1 / d}$ so that $L^{d}=2$. Then $L^{d-2}=2^{1-2 / d}$ and this model has been considered by Bleher and Major for $d>2$. They established that there exists spontaneous magnetization if $d>2$ and the inverse temperature is sufficiently large [7]. The parameter $2^{1-2 / d}$ is written $c$ in [7].

\section{Recursion Formulas and Probabilistic A Priori Bounds}

Our recursion formulas for the hierarchical system are now:

$$
\begin{aligned}
\mu_{n}(\phi)= & \left(\mathscr{Z}_{n}\right)^{-1} \exp \left[-\sum_{k=0}^{K-n} \sum_{x \in \Lambda_{n+k}} H\left(\left(C^{k} \vec{\phi}\right)\left(y_{1}\right), \ldots,\left(C^{k} \vec{\phi}\left(y_{r}\right)\right)\right] \prod_{x \in \Lambda_{n}} g_{n}\left(\vec{\phi}^{2}(x)\right),\right. \\
g_{n}\left(\varphi^{2}\right)= & \left(\mathscr{N}_{n}\right)^{-1} \int \delta\left[\vec{\varphi}-L^{-2} \sum \vec{\phi}\left(y_{i}\right)\right] \exp \left[-H\left(\vec{\phi}\left(y_{1}\right), \ldots, \vec{\phi}\left(y_{r}\right)\right)\right] \\
& \cdot \prod_{i=1}^{r} g_{n-1}\left(\vec{\phi}^{2}\left(y_{i}\right)\right) d \vec{\phi}\left(y_{i}\right),
\end{aligned}
$$

where $r=L^{2},\left\{\mathscr{Z}_{n}\right\}$ and $\left\{\mathscr{N}_{n}\right\}$ are the normalization constants and we set $\vec{\varphi}=(\varphi, \overrightarrow{0}), \varphi=|\vec{\phi}|>0$ by the rotational invariance.

For simplicity, we assume that $\operatorname{supp} g_{0} \subset[0, \kappa]$ and thus supp $g_{n} \subset[0, \kappa]$ for all $n$. Then $g_{0}(x)=\delta(\kappa-x)$ belongs to this class. This is needed just for the a priori bound discussed below, and it is easy to see that $g_{0}$ can be extended to functions decreasing rapidly for $x=\varphi^{2}>\kappa$.

We first consider model I: using

$$
\begin{aligned}
\sum_{(x, y) \in \square(0)} 2(\vec{\phi}(x)-\vec{\phi}(y))^{2} & =2 L^{2} \sum \vec{\phi}(x)^{2}-2\left[\sum \vec{\phi}(y)\right]^{2} \\
& =2 L^{2} \sum \vec{\phi}(y)^{2}-2 L^{4} \vec{\varphi}^{2},
\end{aligned}
$$

where $\vec{\varphi}={ }^{t}(\varphi, 0, \ldots, 0) \in R^{N}$, we have

$$
\begin{aligned}
g_{n}\left(\varphi^{2}\right) e^{-L^{2} \varphi^{2} / 4} & =\left(\mathcal{N}_{n}\right)^{-1} \int \delta\left[\vec{\varphi}-L^{-2} \sum \vec{\phi}_{i}\right] \prod_{k=1}^{L^{2}} f_{n-1}\left(\varphi^{2}\left(x_{k}\right)\right), \\
f_{n-1}\left(\varphi^{2}\right) & =g_{n-1}\left(\varphi^{2}\right) \exp \left[-\varphi^{2} / 4\right] .
\end{aligned}
$$

Thus $g_{n}\left(\varphi^{2}\right)$ is the probability density (except for $\left.\exp \left(-L^{2} \varphi^{2} / 4\right)\right)$ that the average of $L^{2}$ spins with distribution $f_{n-1}\left(\varphi^{2}\right)$ takes its value at $\vec{\phi}($ or $(\varphi, 0))$. The correction 
term $\exp \left[\left(L^{2} \varphi^{2}-\sum \phi^{2}(x)\right) / 4\right]$ comes from the Laplacian part, and enhances the probability that $\varphi$ takes larger values.

For $N \geqq 3$ it is enough to set $L^{2}=2$, and we have:

$$
\begin{aligned}
g_{n}\left(\varphi^{2}\right) & =\left(\mathscr{N}_{n}\right)^{-1} e^{-\varphi^{2} / 2} \int \delta\left[\vec{\varphi}-\frac{1}{2}\left(\vec{\phi}_{1}+\vec{\phi}_{2}\right)\right] \prod f_{n-1}\left(\phi_{i}^{2}\right) d \vec{\phi}_{i} \\
f_{n-1}\left(\varphi^{2}\right) & =g_{n-1}\left(\varphi^{2}\right) e^{-\varphi^{2} / 4} .
\end{aligned}
$$

Theorem 1 ( $A$ priori bound for 2 balls.) Assume $N \geqq 3$. Then for any $f_{n-1}(x) \geqq 0$ such that supp $f_{n-1} \subset[0, \kappa], g_{n}(x)$ is monotone decreasing in $x$ such that $x \in[\kappa / 4, \kappa]$. If $N \geqq 4$, then $g_{n}(x)$ satisfies the following a priori bound for $x \in[\kappa / 4, \kappa]$ :

$$
-\left[\ln g_{n}(x) e^{-x / 2}\right]^{\prime} \equiv \frac{\partial}{\partial x} V_{n}(x)+\frac{1}{2} \geqq \frac{1}{2 x}+\frac{k}{\kappa-x},
$$

where $k \equiv(N-3) / 2$.

Proof. By the rotational invariance, set $\vec{\phi}_{1}=(\varphi+s, \vec{u})$ and $\vec{\phi}_{2}=(\varphi-s,-\vec{u})$ $\left(s \in R, \vec{u} \in R^{N-1}\right)$ to find that

$$
\begin{aligned}
g_{n}(x) e^{-x / 2} & =\text { const } \int d s \int u^{N-2} d u f_{n-1}\left((\varphi+s)^{2}+u^{2}\right) f_{n-1}\left((\varphi-s)^{2}+u^{2}\right) \\
& =\frac{\text { const }}{\sqrt{x}} \int_{0}^{\kappa} \int_{0}^{\kappa} d p d q \theta(\mu(p, q ; x))[\mu(p, q ; x)]^{k} f_{n-1}(p) f_{n-1}(q)
\end{aligned}
$$

by a trivial change of variables, where

$$
\begin{aligned}
\mu(p, q ; x) & =\frac{p+q}{2}-x-\frac{(p-q)^{2}}{16 x}, \\
\theta(\mu) & =\begin{array}{l}
1, \quad \text { if } \mu \geqq 0 \\
0, \quad \text { otherwise }
\end{array} \\
k & =(N-3) / 2 .
\end{aligned}
$$

Now $p$ and $q \in[0, \kappa]$, and $\mu(p, q ; x)$ is decreasing in $x>|p-q| / 4$. Thus so are $\theta(\mu(p, q ; x))$ and $[\mu(p, q ; x)]^{k}$. Since $\kappa \geqq|p-q|$, the former half of the theorem is clear. Next for $N \geqq 4$,

$$
\begin{aligned}
-\left[\ln g_{n}(x) e^{-x / 2}\right]^{\prime} & \equiv V_{n}(x)^{\prime}+1 / 2 \\
& =\frac{1}{2 x}+k \frac{\int_{D} d p d q\left\{\frac{1-(p-q)^{2} / 16 x^{2}}{\mu(p, q ; x)}\right\}[\mu(p, q ; x)]^{k} f_{n-1}(p) f_{n-1}(q)}{\int_{D} d p d q[\mu(p, q ; x)]^{k} f_{n-1}(p) f_{n-1}(q)} \\
& \geqq \frac{1}{2 x}+k \inf _{D} \frac{1-\frac{(p-q)^{2}}{16 x^{2}}}{\mu(p, q ; x)}
\end{aligned}
$$

where

$$
\begin{aligned}
D & =\left\{(p, q) \in[0, \kappa]^{2}: \mu(p, q ; x) \geqq 0,0 \leqq q, p \leqq \kappa\right\} \\
& =\left\{(p, q) \in[0, \kappa]^{2} ;(2 \sqrt{x}-\sqrt{p})^{2} \leqq q \leqq(2 \sqrt{x}+\sqrt{p})^{2}\right\}
\end{aligned}
$$


(see Fig. 1a in Sect. 4 in advance). $1-(p-q)^{2} / 16 x^{2}$ is positive if $x \geqq \kappa / 4$ and the infimum of (3.7b) is taken by $p=q=\kappa$. Q.E.D.

For $N<4$, we improve the bounds by taking larger blocks $(L=2)$.

Theorem 2 ( $A$ priori bound for 4 balls.) Set $L=2$ and $N>5 / 3$. Then for any $f_{n-1}(x) \geqq 0$ such that supp $f_{n-1} \subset[0, \kappa], g_{n}(x) e^{-x}$ is decreasing in $x \in[\kappa / 4, \kappa]$ and satisfies the following bounds:

$$
-\left[\ln g_{n}(x) e^{-x}\right]^{\prime} \geqq \begin{array}{ll}
\frac{1}{2 x}+\frac{\tilde{k}}{\kappa-x}, & \text { for } \quad N \geqq 3, \\
C_{1} \frac{1}{2 x}+C_{2} \frac{\tilde{k}}{\kappa-x}, & \text { for } \quad N=2 .
\end{array}
$$

Here $\tilde{k}=(3 N-5) / 2$, and $C_{i} \gtrsim 1$ uniformly in $x \in[\kappa / 4, \kappa]$.

Proof. It is enough to assume $N=2$ or $N=3$. For $L=2$, we have

$$
\begin{aligned}
g_{n}(x) e^{-x}= & \int d \vec{\Phi}_{1} d \vec{\Phi}_{2} \delta\left[\vec{\Phi}-\frac{1}{2}\left(\vec{\Phi}_{1}+\vec{\Phi}_{2}\right)\right] \\
& \cdot \int d \vec{\phi}_{1} d \vec{\phi}_{2} \delta\left[\vec{\Phi}_{1}-\frac{1}{2}\left(\vec{\phi}_{1}+\vec{\phi}_{2}\right)\right] f_{n-1}\left(\vec{\phi}_{1}^{2}\right) f_{n-1}\left(\vec{\phi}_{2}^{2}\right) \\
& \cdot \int d \vec{\phi}_{3} d \vec{\phi}_{4} \delta\left[\vec{\Phi}_{2}-\frac{1}{2}\left(\vec{\phi}_{3}+\vec{\phi}_{4}\right)\right] f_{n-1}\left(\vec{\phi}_{3}^{2}\right) f_{n-1}\left(\vec{\phi}_{4}^{2}\right) \\
= & \int \prod d p_{i} K_{N}\left(\left\{p_{i}\right\}, x\right) \prod f_{n-1}\left(p_{i}\right)
\end{aligned}
$$

by introducing radial variables $p_{i}=\vec{\phi}_{i}^{2}(i=1, \ldots, 4)$. Here by putting $p=\vec{\Phi}_{1}^{2}$ and $q=\vec{\Phi}_{2}^{2}$, the kernel $K_{N}$ has the expression

$$
\begin{aligned}
& \int_{\mathscr{D}} d p d q \mathscr{K}_{N}\left(\left\{p_{i}\right\} ; p, q, x\right), \\
& \mathscr{K}_{N} \equiv \frac{1}{\sqrt{p q x}}\left[\mu(p, q: x) \mu\left(p_{1}, p_{2} ; p\right) \mu\left(p_{3}, p_{4} ; q\right)\right]^{k} .
\end{aligned}
$$

The region $\mathscr{D}$ is the set of $(p, q) \in[0, \kappa]^{2}$ in which all $\mu$ 's are positive: $\mathscr{D}=D \cap \Delta$, where $D$ is given by (3.8) and

$$
\Delta=\left\{(p, q) ;\left(\sqrt{p_{1}}-\sqrt{p_{2}}\right)^{2} \leqq 4 p \leqq\left(\sqrt{p_{1}}+\sqrt{p_{2}}\right)^{2},\left(p \rightarrow q, p_{i} \rightarrow p_{i+2}\right)\right\} .
$$

Obviously $\mathscr{D}=\phi$ if $\sum \sqrt{p_{i}}<4 \sqrt{x}$ and $\Delta$ is not in $D$ if $x>\kappa / 4$, and only one corner of $\Delta$ is $\in D$ if $x>(3 / 4)^{2} \kappa$.

For $N=3$, the $K_{N}$ is explicitly obtained:

$$
K_{3}=\frac{\text { const }}{\sqrt{x}}\left\{\begin{array}{ll}
{\left[\left(\sum \sqrt{p_{i}}\right)-4 \sqrt{x}\right]^{2},} & x \in\left[(3 / 4)^{2} \kappa, \kappa\right] \\
\sqrt{p_{1}}\left(\sum_{i \neq 1} \sqrt{p_{i}}-4 \sqrt{x}\right), & x \in\left[\kappa / 4,(3 / 4)^{2} \kappa\right]
\end{array},\right.
$$

where we assumed that $p_{1}=\min \left\{p_{i}\right\}_{1}^{4}$ in the second equation.

For $N=2$, we have $\mu$ 's include $(p q x)^{-1 / 2}$ in Eq. (3.11b) and set $\mu(p, q ; x) \equiv$ $8 x(p+q)-x^{2}-(p-q)^{2}$. We furthermore set $p=p_{0}-\zeta, q=q_{0}-\xi(\zeta, \xi \geqq 0)$ with $p_{0}=\left(\sqrt{p_{1}}+\sqrt{p_{2}}\right)^{2} / 4$ and $q_{0}=\left(\sqrt{p_{3}}+\sqrt{p_{4}}\right)^{2} / 4$, and define

$$
\sigma(x, \xi)=(2 \sqrt{x}+\sqrt{q})^{2}-p_{0}, \quad \tau(x, \xi)=p_{0}-(2 \sqrt{q}-\sqrt{x})^{2} .
$$


Thus we have

$$
\begin{aligned}
\mu(p, q ; x) & =[\sigma(x, \xi)+\zeta][\tau(x, \xi)-\zeta] \\
\mu\left(p_{1}, p_{2} ; p\right) & =\zeta\left[\sqrt{p_{1} p_{2}}-\zeta\right], \quad \mu\left(p_{3}, p_{4} ; q\right)=\xi\left[\sqrt{p_{3} p_{4}}-\xi\right] .
\end{aligned}
$$

Introduce two parameters $s \in[0,1]$ and $t \in[0,1]$, and let $\zeta=\tau(x, \xi) t$ and $\xi=\xi_{\max } s$, where $\xi_{\max }=q_{0}-\left(2 \sqrt{x}-\sqrt{p_{0}}\right)^{2}$. Then we have

$$
\begin{aligned}
K_{N=2}= & \xi_{\max }^{1 / 2} \int_{0}^{1} d s \int_{0}^{1} d t \frac{1}{[s t]^{1 / 2}} I(x ; s, t), \\
I(x ; s, t)= & \left\{\left[\sqrt{p_{1} p_{2}}-\tau\left(x, \xi_{\max } s\right) t\right]\left[\sqrt{p_{3} p_{4}}-\xi_{\max } s\right]\right. \\
& \left.\cdot\left[\sigma\left(x, \xi_{\max } s\right)+\tau\left(x, \xi_{\max } s\right) t\right]\right\}^{-1 / 2} .
\end{aligned}
$$

$\xi_{\max }$ is monotone decreasing in $x \in[\kappa / 4, \kappa]$ and so is $\tau\left(x, \xi_{\max } s\right)=p_{0}-(2 \sqrt{x}-$ $\left.\left(q_{0}-\xi_{\max } s\right)^{1 / 2}\right)^{2}$. Moreover

$$
\sigma\left(x, \xi_{\max } s\right)+\tau\left(x, \xi_{\max } s\right) t=\left[4 x-p_{0}+q_{0}-\xi_{\max } s\right](1-t)+4 \sqrt{x} \sqrt{q_{0}-\xi_{\max } s}(1+t)
$$

is monotone increasing. Thus $I(x ; s, t)$ is monotone decreasing for any $s$ and $t$. Thus $K_{N=2}$ decreases faster than $\xi_{\max }$. By symmetry $K_{2}$ decreases faster than $\left[p_{0}-\left(2 \sqrt{x}-\sqrt{q_{0}}\right)^{2}\right]^{1 / 2}$ and thus faster than

$$
\begin{aligned}
& \left\{\left[q_{0}-\left(2 \sqrt{x}-\sqrt{q_{0}}\right)^{2}\right]\left[p_{0}-\left(2 \sqrt{x}-\sqrt{q_{0}}\right)^{2}\right]\right\}^{1 / 4} \\
& \quad=\left[\sqrt{p_{0}}+\sqrt{q_{0}}-2 \sqrt{x}\right]^{1 / 2}\left[4 x-\left(\sqrt{p_{0}}-\sqrt{q_{0}}\right)^{2}\right]^{1 / 4} .
\end{aligned}
$$

For $x \in\left[\kappa / 4,(3 / 4)^{2} \kappa\right]$, we assume $p_{1}=\min \left\{p_{i}\right\}_{1}^{4}$ and set $p=p_{0}-\zeta$ and $q=q_{0}-\xi$. Then $\zeta \in\left[0, \sqrt{p_{1} p_{2}}\right]$, and $\xi \in\left[0, q_{0}-\left(2 \sqrt{x}-\sqrt{p_{0}-\zeta}\right)^{2}\right] \equiv\left[0, \xi_{\max }(\zeta)\right]$. Set $\zeta_{\max }=$ $\sqrt{p_{1} p_{2}}$ and $\xi=\xi_{\max } t$. Thus we have

$$
\begin{aligned}
K_{N=2} & =\int_{0}^{\zeta_{\max }} d \zeta \frac{1}{\sqrt{\zeta\left(\zeta_{\max }-\zeta\right)}} \int_{0}^{1} d t \frac{1}{\sqrt{t(1-t)}} J(t, \zeta ; x), \\
J(t, \zeta ; x) & =\left[\sqrt{p_{3} p_{4}}-\xi_{\max } t\right]^{-1 / 2}\left[(2 \sqrt{x}+\sqrt{p})^{2}-q_{0}+\xi_{\max } t\right]^{-1 / 2},
\end{aligned}
$$

and thus

$$
\begin{aligned}
-\left[\ln K_{N=2}\right]^{\prime} & >\inf _{t, \zeta}-[\ln J(t, \zeta ; x)]^{\prime} \\
& =\frac{1}{2} \inf \left[\frac{\left[4-2 \frac{\sqrt{p}}{\sqrt{x}}\right] t}{\sqrt{p_{3} p_{4}}-\xi_{\max } t}+\frac{4(1-t)+2 \frac{\sqrt{p}}{\sqrt{x}}(1+t)}{\left(4 x-q_{0}+p\right)(1-t)+4 \sqrt{x p}(1+t)}\right] \\
& \geqq \frac{1}{4 x},
\end{aligned}
$$

using $\sum B_{i} / \sum A_{i} \geqq \min \left\{B_{i} / A_{i}\right\}$ in the second line. Q.E.D. 
Finally we consider model II. Again set $L=2(r=4)$ and $\vec{\varphi}=(\varphi, 0)$.

$$
\begin{aligned}
g_{n}(x)= & \int \delta\left[\vec{\varphi}-\left(\vec{\Phi}_{1}+\vec{\Phi}_{2}\right) / 2\right] d \vec{\Phi}_{1} d \vec{\Phi}_{2} \\
& \cdot \int \Pi d \vec{\phi}_{i} \delta\left[\vec{\Phi}_{1}-\left(\vec{\phi}_{1}+\vec{\phi}_{2}\right) / 2\right] \delta\left[\vec{\Phi}_{2}-\left(\vec{\phi}_{3}+\vec{\phi}_{4}\right) / 2\right] \\
& \cdot \exp \left[-H\left(\vec{\phi}_{1}, \ldots, \vec{\phi}_{4}\right)\right] \Pi g_{n-1}\left(\vec{\phi}_{i}^{2}\right) .
\end{aligned}
$$

Set $\vec{\Phi}_{i}={ }^{t}(\varphi \pm s, \pm \vec{\omega} u)(+$ for $i=1,-$ for $i=2$ and $|\vec{\omega}|=1)$ and insert $1=\int d p d q \delta[p-$ $\left.(\varphi+s)^{2}-u^{2}\right] \delta\left[q-(\varphi-s)^{2}-u^{2}\right]$. Define the orthogonal matrices $R_{1}$ and $R_{2}$ by $R_{i} \vec{\Phi}_{i}=\left(\varphi_{i}, 0\right)$, where $\varphi_{1}=\sqrt{p}$ and $\varphi_{2}=\sqrt{q}$. Then

$$
\begin{aligned}
g_{n}(x)= & \int d \vec{\omega} \int_{D} d p d q \frac{\mu(p, q ; x)^{k}}{\sqrt{x}}\left\{\int \pi d \vec{\phi}_{i} g_{n-1}\left(\vec{\phi}_{i}^{2}\right) \delta\left[(\sqrt{p}, \overrightarrow{0})-\frac{1}{2} \sum_{1}^{2} \vec{\phi}_{i}\right]\right. \\
& \left.\cdot \delta\left[(\sqrt{q}, \overrightarrow{0})-\frac{1}{2} \sum_{3}^{4} \vec{\phi}_{i}\right] \exp \left[-H\left(R_{1} \vec{\phi}_{1}, \ldots, R_{2} \vec{\phi}_{4}\right)\right]\right\} .
\end{aligned}
$$

We again set $\vec{\phi}_{i}=\left(\sqrt{p} \pm s_{1}, \pm \vec{\omega}_{1} u_{1}\right)(+$ for $i=1,-$ for $i=2)$ and $\vec{\phi}_{j}=\left(\sqrt{q} \pm s_{2}\right.$, $\left.\pm \vec{\omega}_{2} u_{2}\right)(+$ for $j=3$, - for $j=4)$, and insert

$$
1=\int\left(\Pi d p_{i}\right) \delta\left[p_{1}-\left(\sqrt{p}+s_{1}\right)^{2}-u_{1}^{2}\right] \cdots \delta\left[p_{4}-\left(\sqrt{q}-s_{2}\right)^{2}-u_{2}^{2}\right] .
$$

We thus explicitly have

$$
\begin{aligned}
& g_{n}(x)= \int d \vec{\omega} \Pi d \vec{\omega}_{i} \int \Pi d p_{i} g_{n-1}\left(p_{i}\right) \int d p d q \mathscr{K}_{N}\left(\left\{p_{i}\right\} ; p, q ; x\right) \\
& \cdot \exp \left[-H\left(R_{1} \vec{\phi}_{1}, \ldots, R_{2} \overrightarrow{\mathscr{\phi}}_{4}\right)\right], \\
& \vec{\phi}_{1}={\sqrt{p_{1}}}^{t}\left(\left[1-\frac{\mu\left(p_{1}, p_{2} ; p\right)}{p_{1}}\right]^{1 / 2}, \vec{\omega}_{1} \frac{\sqrt{\mu\left(p_{1}, p_{2} ; p\right)}}{\sqrt{p_{1}}}\right) \\
& \vec{\phi}_{1}^{2}=p_{i} \quad(i=1, \ldots, 4)
\end{aligned}
$$

and etc. Note that only $\mathscr{K}_{N}$ and $R_{i}$ depend on $x$. By (2.9a)

$$
H\left(R_{1} \vec{\phi}_{1}, R_{1} \vec{\phi}_{2}, R_{2} \vec{\phi}_{3}, R_{2} \vec{\phi}_{4}\right)=H\left(\vec{\phi}_{1}, \vec{\phi}_{2}, R \vec{\phi}_{3}, R \vec{\phi}_{4}\right), \quad R=R_{1}^{-1} R_{2}
$$

Let $R=1+\delta R$. Then we have:

$$
\delta R=\frac{1}{\sqrt{p q}}\left(\begin{array}{cccc}
2 x-(\sqrt{p}-\sqrt{q})^{2} & 2 \sqrt{x} u & \sqrt{q}\left(\vec{\omega}, u_{3}\right) u & . \\
2 \sqrt{x} u & -2 x+(\sqrt{p}-\sqrt{q})^{2} & \sqrt{q}\left(\vec{\omega}, \vec{u}_{3}\right)(\varphi+s) & . \\
-\sqrt{p}\left(\vec{\omega}, \vec{u}_{3}\right) u & \sqrt{p}\left(\vec{\omega}, \vec{u}_{3}\right)(\varphi-s) & 0 & . \\
\vdots & \vdots & 0 & .
\end{array}\right)
$$

where $\vec{\omega}, \vec{u}_{3}, \vec{u}_{4}, \ldots, \vec{u}_{N}\left(\in R^{N-1}\right)$ are orhogonal unit vectors. So $\left|\partial\left(\delta R_{i i}\right) / \partial x\right|$ are bounded by $2(p q)^{-1 / 2}$ and terms containing $(\varphi \pm s)$ are also bounded by $O\left(p^{-1}\right)$ or by $O\left(q^{-1}\right)$. Risky terms containing $u=\sqrt{\mu}$ are off-diagonal, and connecting $\left(\phi_{i}\right)_{1}$ and $\left(\phi_{j}\right)_{2}$ or $\left(\phi_{i}\right)_{1,2}$ and $\left(\phi_{j}\right)_{\ell}(\ell \geqq 3)$. Then singular terms of $\mu^{-1 / 2}$ do not appear in $\partial H\left(\phi_{1}, \phi_{2}, R \phi_{3}, R \phi_{4}\right) / \partial x$ after integrating over $\vec{\omega}$ and $\vec{\omega}_{i}$ 's. Thus by taking the derivative of $(3.17 \mathrm{a})$

Theorem 3. If $H\left(\left\{\vec{\phi}_{i}\right\}\right)$ satisfies the conditions $(2.9 a, b)$ then $\left\{g_{n+1}(x)\right\}$ again satisfy 
the a priori bound for $x \in[\kappa-\delta, \kappa]$ :

$$
-\left[\ln g_{n+1}(x)\right]^{\prime} \geqq \operatorname{const} \frac{\tilde{k}}{\kappa-x} \text {. }
$$

\section{Trajectories of $\left\{g_{n}(x)\right\}$; (I) Large Field Region}

Throughout Sects. 4,5 and 6, we assume $L^{2} \geqq 2, N>3$ and $\kappa>O\left((N-3)^{2}\right)+1$ without loss of generality. Let $k \equiv \frac{1}{2}(N-3)$ and define

$$
\begin{aligned}
& A=\int_{D} d p d q \mu(p, q ; x)^{k} \theta(\mu) f_{n}(p) f_{n}(q), \\
& B=\int_{D} d p d q\left[1-\frac{(p-q)^{2}}{16 x^{2}}\right] \mu(p, q ; x)^{k-1} \theta(\mu) f_{n}(p) f_{n}(q) .
\end{aligned}
$$

We introduce a strictly positive constant $\omega=$ const $k$ and let

$$
\kappa_{n}=\kappa-n \omega, \quad n=0,1, \ldots, N_{0},
$$

where $N_{0}$ is defined so that $\kappa_{N_{0}}=O\left(k^{2}\right)$. Then the behaviors of $\left\{g_{n}\right\}$ are classified into three regions:

(1) scaling region: $n=0,1, \ldots, N_{0}$,

(2) transitive region: $n=N_{0}, N_{0}+1, \ldots, N_{0}^{\prime}=N_{0}+O\left(k^{2}\right)$,

(3) high-temperature region: $n>N_{0}^{\prime}$.

Theorem 4. Assume that $\kappa \gg k=(N-3) / 2 \geqq 1 / 2$. Then there exist a constant $\omega=C_{1} k$ and poisitive increasing and convex functions $\left\{\alpha_{n}(x) ; n=1,2, \ldots, N_{0}\right\}$, where $N_{0}$ is the largest integer less than $\left(\kappa-O\left(k^{2}\right)\right) / \omega$ and each $\alpha_{n}(x)$ is defined on $I_{n}=\left[\kappa_{n}, \kappa\right], \kappa_{n}=\kappa-n \omega$, and satisfies:

(1) $-\left[\ln g_{n}(x)\right]^{\prime} \geqq \alpha_{n}(x), \quad x \in I_{n}$,

(2) $\alpha_{n}(x) \geqq\left[2-\delta_{k}\right] \alpha_{n-1}(x)$, for $x \in I_{n-1}$,

(3) $\left[2-\delta_{k}\right] \alpha_{\min } \geqq \alpha_{n}(x) \geqq \alpha_{\min }$ for $x \in\left[\kappa_{n}, \kappa_{n-1}\right]$,

(4) $\alpha_{n}\left(\kappa_{n}\right)=\alpha_{\min } \geqq 2$.

Here $\delta_{k} \ll 1, \delta_{k}<$ const $/ k$ and $\left(2-\delta_{k}\right)$ can be made arbitrarily close to 2 by choosing $\omega$ small. Moreover there exists $\varepsilon_{k} \ll 1$ such that

$$
2 \omega \alpha_{\min } \leqq k\left(1-\varepsilon_{k}\right) .
$$

If $x \in\left[\kappa_{n-n_{0}}, \kappa_{n-n_{0}-1}\right]$, then the $\delta_{k}$ in $(4.4 \mathrm{~b})$ can be replaced by

$$
\delta_{k}^{n}(x)<\text { const } 2^{n_{0}-n} \text {. }
$$

Remark 2. $\alpha_{\min }$ will be chosen so that

$$
\left[2-\tilde{\delta}_{k}\right]\left[\alpha_{\min }+\frac{1}{4}\right]-\frac{1}{2}>\alpha_{\min }
$$

for some $\tilde{\delta}_{k}<\delta_{k}$. Then $\delta_{k} \rightarrow \tilde{\delta}_{k}$ as $\alpha_{\min } \rightarrow \infty$ and this yields the lower bound about $1 / 4$ for $\alpha_{\min }$.

We use the following technical lemma (see the appendix): 
Lemma 5. Let $f(\zeta)$ be a positive function defined on $R_{+}$. Let

$$
\left\langle\zeta^{m}\right\rangle(\lambda, s, t, k-1, f)=\frac{\int_{0}^{\lambda} d \zeta \zeta^{m}\left(s+\zeta-t \zeta^{2}\right)^{k-1} \zeta^{\ell} f(\zeta)}{\int_{0}^{\lambda} d \zeta\left(s+\zeta-t \zeta^{2}\right)^{k-1} \zeta^{\ell} f(\zeta)},
$$

where $m, \lambda, s, t, k-1$ and $\ell$ are positive and $s+\zeta-t \zeta^{2} \geqq 0$ for $\zeta \in[0, \lambda]$. Then $\left\langle\zeta^{m}\right\rangle(\lambda, s, t, k-1, f)$ is monotone increasing in $\lambda$, and decreasing in $s$ and $t$. If $-[\ln f(\zeta)]^{\prime} \geqq-[\ln g(\zeta)]^{\prime}(g(\zeta)>0)$,

$$
\left\langle\zeta^{m}\right\rangle(\lambda, s, t, k-1, f) \leqq\left\langle\zeta^{m}\right\rangle(\lambda, s, t, k-1, g) .
$$

Thus if $-[\ln f(\zeta)]^{\prime} \geqq \alpha(>0)$, the upper bound is realized by $e^{-\alpha \zeta}$.

Proof of Theorem 4. For $n=1$, this is trivial from Theorem 1:

$$
\alpha_{1}(x) \geqq\left\{\frac{1}{2 x}+\frac{k}{\kappa-x}\right\}-\frac{1}{2}, \quad x>\frac{\kappa}{4} \text {. }
$$

Then set $\kappa_{1}=\kappa-C_{1} k$, where $C_{1} \leqq 1 / 2$ (later we choose $\left.C_{1} \ll 1\right)$. It is also clear that $\alpha_{1}(x)$ can be chosen to be convex and increasing in the region $I_{1}=[\kappa-\omega, \kappa]$.

Assume that the theorem holds for $1, \ldots, n$. Then for $n+1$, we encounter three cases depending on the magnitudes of $x=\Phi^{2}$ :

(1) $x \in\left[\kappa_{n}, \kappa_{n-\tilde{n}}\right]$, transitive region,

(2) $x \in\left[\kappa_{n+1}=\kappa_{n}-\omega, \kappa_{n}\right]$, small field region,

(3) $x>\kappa_{n-\tilde{n}}$, large field region,

where $\tilde{n} \geqq 1$ is a suitable small integer.

(case 1) $x=\varphi^{2} \in\left[\kappa_{n}, \kappa_{n-\tilde{n}}\right]$ :

Decompose $D$ into two regions $D_{1}$ and $D_{2}$ (see Fig. 1a):

$$
\begin{aligned}
& D_{1}=\{(p, q) \in D ; x \leqq q \leqq p \leqq \kappa\} \\
& D_{2}=\{(p, q) \in D ; 0 \leqq q \leqq x\} .
\end{aligned}
$$

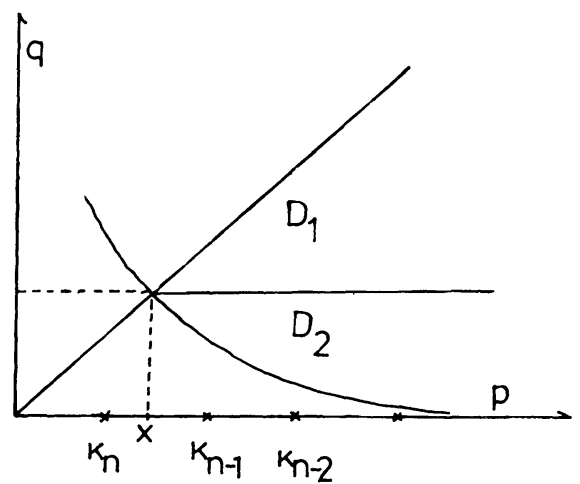

Fig. 1a. Domain $D=\left\{(p, q) \in[0, \kappa]^{2} ; \mu(p, q ; x) \geqq 0\right\}$ and its decomposition into $D_{1}, D_{2}$ By the $p \leftrightarrow q$ symmetry it suffices to consider the region $q<p$ 
Let

$$
\begin{aligned}
& A_{i}=\int_{D_{i}} d p d q \mu(p, q ; x)^{k} f_{n}(p) f_{n}(q) d p d q \\
& B_{i}=\int_{D_{i}} d p d q\left[1-\frac{(p-q)^{2}}{16 x^{2}}\right] \mu(p, q ; x)^{k-1} f_{n}(p) f_{n}(q) d p d q
\end{aligned}
$$

Then we have

$$
k \frac{B}{A}=\frac{A_{1}}{A}\left(k \frac{B_{1}}{A_{1}}\right)+\frac{A_{2}}{A}\left(k \frac{B_{2}}{A_{2}}\right) \geqq \min \left\{k \frac{B_{i}}{A_{i}}\right\} .
$$

Lemma 6. Let $k>0$. Then

(1) $k \frac{B_{2}}{A_{2}} \geqq 2\left[\alpha_{n}(x)+\frac{1}{4}\right]-\frac{1}{2 x}$

(2) $k \frac{B_{1}}{A_{1}} \geqq \frac{2 k}{k+1}\left[\alpha_{n}(x)+\frac{1}{4}\right]$ for $k \geqq 1$,

$$
k \frac{B_{1}}{A_{1}} \geqq \frac{1}{2}\left(1-O\left(x^{-1}\right)\right)\left[\alpha_{n}(x)+\frac{1}{4}\right] \text { for } k=1 / 2 .
$$

Proof. (1) First note that

$$
\left(1-(p-q)^{2} / 16 x^{2}\right) k \mu(p, q ; x)^{k-1}=\left(1+\frac{p-q}{4 x}\right) 2\left(\partial \mu^{k} / \partial p\right) .
$$

Then the left-hand side of Eq. (4.9) is equal to

$$
\frac{\int_{\text {min }}^{x} d q f_{n}(q) \int_{p_{\min }}^{p_{\max }} d p\left[\frac{\partial}{\partial p} \mu^{k}\right] 2\left(1+\frac{p-q}{4 x}\right) f_{n}(p)}{\int_{q_{\min }}^{x} d q f_{n}(q) \int_{p_{\min }}^{p_{\max }} d p \mu^{k} f_{n}(p)}
$$

where $p_{\min }=p_{\min }(q)=(2 \sqrt{x}-\sqrt{q})^{2} \geqq x, p_{\max }=\min \left\{\kappa,(2 \sqrt{x}+\sqrt{q})^{2}\right\}$, and $q_{\min }=0$ if $x<\kappa / 4$ and $q_{\min }=(2 \sqrt{x}-\sqrt{\kappa})^{2}$ otherwise. Therefore using the integration by parts and the fact that $\mu(p, q ; x)^{k} f_{n}(p)$ vanishes at $p_{\max }$ and $p_{\min }$, one obtains

$$
\begin{aligned}
\text { L.H.S. of Eq. (4.7) } & \geqq-\frac{1}{2 x}+\inf _{p \geqq x} 2(\partial / \partial p)\left[-\ln f_{n}(p)\right] \\
& \geqq-\frac{1}{2 x}+2\left[\alpha_{n}(x)+\frac{1}{4}\right] .
\end{aligned}
$$

(2) Set $p=x+\zeta$ and $q=x+\xi$. Then for $k \geqq 1$,

where

$$
k \frac{B_{1}}{A_{1}}=2 k \frac{1-\frac{\langle\langle\varepsilon\rangle\rangle}{2 x}}{\langle\langle\zeta+\xi\rangle\rangle-\langle\langle\varepsilon\rangle\rangle} \geqq \frac{2 k}{\langle\langle\zeta+\xi\rangle\rangle},
$$

$$
\left\langle\langle\cdot\rangle=\mathscr{N}^{-1} \int d \zeta d \xi(\cdot) \mu(x+\zeta, x+\xi ; x)^{k-1} f_{n}(x+\zeta) f_{n}(x+\xi)\right.
$$


and $\varepsilon \equiv(\zeta-\xi)^{2} / 8 x$. Then $\langle\langle\varepsilon\rangle\rangle=O\left(\alpha^{-2} x^{-1}\right)$. To evaluate $\langle\langle\cdot \cdot\rangle$, set $\zeta=r \cos (\theta) /$ $\sqrt{2} \cos (\theta-\pi / 4)$ and $\xi=r \sin (\theta) / \sqrt{2} \cos (\theta-\pi / 4)$ with the Jacobian $r / 2 \cos ^{2}(\theta-\pi / 4)$ $(0<\theta<\pi / 2)$. Apply Lemma 5 for each $\theta$ :

$$
\begin{aligned}
\langle\langle\zeta+\xi\rangle\rangle & \leqq \sup _{\theta} \frac{\int d r r^{k+1} F_{\theta}(r)}{\int d r r^{k} F_{\theta}(r)} \leqq \frac{k+1}{\alpha+1 / 4}, \\
F_{\theta}(r) & \equiv f_{n}\left(x+\frac{r \cos (\theta)}{\sqrt{2} \cos (\theta-\pi / 4)}\right) f_{n}\left(x+\frac{r \sin (\theta)}{\sqrt{2} \cos (\theta-\pi / 4)}\right) .
\end{aligned}
$$

For $k=1 / 2$ one easily finds:

$$
k \frac{B_{1}}{A_{1}} \geqq\left(1-O\left(x^{-1}\right)\right) \frac{2 k}{\langle\langle\zeta+\xi\rangle\rangle},
$$

where $\langle\langle\cdot\rangle\rangle$ is defined here by setting $\mu=(\zeta+\xi) / 2$. Use the previous method.

Q.E.D.

For $k=1$ and $1 / 2$, we need to calculate $k B_{1} / A_{1}$ a bit carefully by taking the smallness of $\omega$ and $\alpha_{\min } \omega$ into consideration. Assume that our assumption holds for $0,1, \ldots, n$. Then for $x \in\left[\kappa_{n}, \kappa_{n-\tilde{n}}\right](\tilde{n}>1)$ and for $\zeta>0$, we have

$$
\left[\ln g_{n}(x+\zeta)\right]^{\prime} \leqq-\exp (C[\zeta / \omega]) \alpha_{n}(x),
$$

where $\left(2-\delta_{k}\right)=\exp (C)$ and $[\zeta / \omega]$ is the largest integer less than or equal to $\zeta / \omega$. This differential inequality implies that

$$
\begin{aligned}
g_{n}(x+\zeta) & \leqq g_{n}(x) \exp \left[-v_{n}(\zeta) \alpha_{n}(x)\right], \\
v_{n}(\zeta) & =\frac{1}{1-\delta_{k}}\left\{e^{C[\zeta / \omega]}-1\right\}+e^{C[\zeta / \omega]}(\zeta-\omega[\zeta / \omega])
\end{aligned}
$$

(including the derivative) and thus

$$
\begin{aligned}
v_{n}(\zeta) \alpha_{n}(x) & =\tilde{\zeta}+\frac{C}{2 \omega \alpha} \tilde{\zeta}^{2}+\cdots \\
\tilde{\zeta} & =\alpha_{n}(x) \zeta .
\end{aligned}
$$

Then if $\omega \alpha$ is small ( $C$ is less than 1$), C / 2 \omega \alpha$ is large and $\langle\langle\zeta+\xi\rangle\rangle$ can be made arbitrarily small, and thus $k B_{1} / A_{1}$ can be made arbitrarily large.

$(k=1)$ The integral (4.12a) is one-dimensional:

$$
\begin{aligned}
k \frac{B_{1}}{A_{1}} & \geqq\left[\frac{\int d \zeta \zeta f_{n}(x+\zeta)}{\int d \zeta f_{n}(x+\zeta)}\right]^{-1} \equiv \frac{1}{\langle\zeta\rangle_{0}} \\
& \geqq\left[\alpha_{n}(x)+1 / 4\right] \frac{\int d \zeta \exp \left[-\zeta-\sigma \zeta^{2}\right]}{\int d \zeta \zeta \exp \left[-\zeta-\sigma \zeta^{2}\right]},
\end{aligned}
$$

where $\sigma=\left[2 \omega \alpha_{n}(x)\right]^{-1}$. This can be made arbitrarily larger than $\alpha_{n}+1 / 4$ by choosing $\omega$ small. (This argument does not work if $\alpha_{n}(x)$ is too large, and then $\tilde{n}$ cannot be too large.) 
$(k=1 / 2)$ We introduce the polar coordinate as before. Then

$$
\begin{aligned}
\frac{1}{2} \frac{B_{1}}{A_{1}} & \geqq\left(1-O\left(x^{-1}\right)\right) \inf _{\theta} \frac{\int d r r^{1 / 2} F_{\theta}(r)}{\int d r r^{3 / 2} F_{\theta}(r)} \\
& \geqq\left(1-O\left(x^{-1}\right)\right)\left[\alpha_{n}(x)+1 / 4\right] \frac{\int d r r^{1 / 2} \exp \left[-r-\sigma r^{2}\right]}{\int d r r^{3 / 2} \exp \left[-r-\sigma r^{2}\right]}
\end{aligned}
$$

and we choose $\alpha_{\min } \omega$ small.

$$
\text { (case 2) } x \in\left[\kappa_{n+1}=\kappa_{n}-\omega, \kappa_{n}\right] \text { : }
$$

We decompose $D$ into four pieces $D_{0}, D_{1}, D_{2}, D_{3}$ (see Fig. 1b):

$$
\begin{aligned}
& D_{0}=\left\{(p, q) \in D ; x \leqq q \leqq \kappa_{n}\right\}, \\
& D_{1}=\left\{(p, q) \in D ; \kappa_{n} \leqq q \leqq p\right\}, \\
& D_{2}=\left\{(p, q) \in D ; 0 \leqq q \leqq\left(2 \sqrt{x}-\sqrt{\kappa_{n}}\right)^{2}\right\}, \\
& D_{3}=\left\{(p, q) \in D ; \kappa_{n} \leqq p,\left(2 \sqrt{x}-\sqrt{\kappa_{n}}\right)^{2}<q<\kappa_{n}\right\} .
\end{aligned}
$$

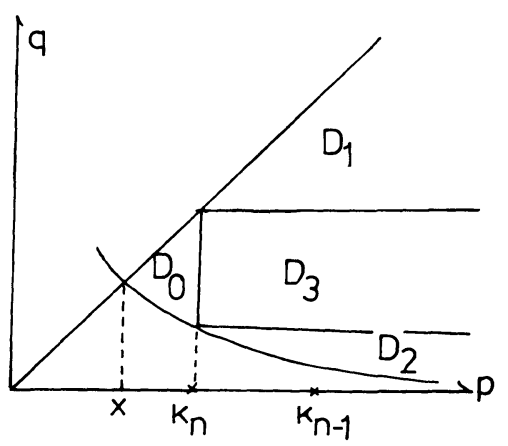

Fig. 1b. Decomposition of $D$ into $D_{0}$ to $D_{3}$. The a priori bound is used to calculate the contribution from $D_{0}$ and the previous methods for the contributions from $D_{1}$ to $D_{3}$

Define $A_{0}$ to $A_{3}$ and $B_{0}$ to $B_{3}$ as before. We already have the a priori bound $k B_{0} / A_{0} \geqq k / \Delta \equiv k /\left(\kappa_{n}-x\right)$ and Lemma $6(1), k B_{2} / A_{2} \geqq-(2 x)^{-1}+2\left(\alpha_{\min }+1 / 4\right)$. We estimate $k B_{i} / A_{i}(i=1,3)$. As for $k B_{1} / A_{1}$,

$$
\begin{aligned}
k \frac{B_{1}}{A_{1}} & =k \frac{\left[1-\left\langle\left\langle(\zeta-\xi)^{2}\right\rangle\right\rangle / 16 x^{2}\right]}{\kappa_{n}-x+\frac{1}{2}\langle\langle\zeta+\xi\rangle\rangle-\left\langle\left\langle(\zeta-\xi)^{2}\right\rangle\right\rangle / 16 x} \\
& \geqq \frac{k}{\kappa_{n}-x+(1 / 2)\langle\langle\zeta+\xi\rangle\rangle},
\end{aligned}
$$

where $\langle\langle\cdot\rangle\rangle$ denotes the expectation value $(4.12 \mathrm{a})$ with the replacements $f_{n}(x+\zeta) \rightarrow$ $f_{n}\left(\kappa_{n}+\zeta\right)$ and $\mu(x+\zeta, x+\xi ; x) \rightarrow \mu\left(\kappa_{n}+\zeta, \kappa_{n}+\xi ; x\right)$. Here $-\left[\ln f_{n}\left(\kappa_{n}+\zeta\right)\right]^{\prime} \geqq \alpha_{n}\left(\kappa_{n}\right)+$ 1/4. Again set $\zeta=r \cos (\theta) / \sqrt{2} \cos (\theta-\pi / 4)$ and etc., and take the maximum over $\theta$. 
Then for $k>1$,

$$
\frac{1}{2}\langle\zeta \zeta+\xi\rangle \leqq \frac{k+1}{2}\left[\alpha_{\min }+1 / 4\right]^{-1},
$$

and for small $k(1 / 2,1)$ we already have:

$$
\frac{1}{2}\left\langle\langle\zeta+\xi\rangle \gg C_{k}\left[\alpha_{\min }+1 / 4\right]^{-1}, \quad C_{k} \ll 1\right.
$$

by choosing $\omega$ small. (This can be applied to any $k$.)

As for $k B_{3} / A_{3}$, if $k \geqq 1$, we have:

$$
\begin{aligned}
k \frac{B_{3}}{A_{3}} & \geqq k \frac{\int_{q_{\min }}^{q_{\max }} f_{n}(q) d q \int_{\kappa_{n}}^{\kappa} d p(1-\varepsilon / x) \mu^{k-1} f_{n}(p) d p}{\int_{q_{\min }}^{q_{\max }} f_{n}(q) d q \int_{\kappa_{n}}^{\kappa} d p \mu^{k} f_{n}(p) d p} \\
& \geqq \inf _{q} \frac{\int_{\kappa_{n}}^{\kappa}(1-\varepsilon / x) \mu(p, q ; x)^{k-1} f_{n}(p) d p}{\int_{\kappa_{n}}^{\kappa} \mu(p, q ; x)^{k} f_{n}(p) d p} \\
& =\inf _{q} \frac{k(1-\langle\varepsilon\rangle / x)}{\frac{1}{2}\left(\kappa_{n}+q\right)-x+\frac{1}{2}\langle\zeta\rangle-\langle\varepsilon\rangle} \\
& \geqq \inf _{q} \frac{2 k}{\left(\kappa_{n}+q-2 x\right)+\langle\zeta\rangle},
\end{aligned}
$$

where $\varepsilon=\left(\kappa_{n}+\zeta-q\right)^{2} / 16 x$ and

$$
\langle\cdot\rangle \equiv \mathscr{N}^{-1} \int_{0}^{\zeta_{\max }}(\cdot) \mu\left(\kappa_{n}+\zeta, q ; x\right)^{k-1} f_{n}\left(\kappa_{n}+\zeta\right) d \zeta .
$$

Thus by Lemma $5,\langle\zeta\rangle$ is maximized when $\mu$ is equal to $\mu\left(\kappa_{n}+\zeta, \kappa_{n} ; \kappa_{n}\right)$ and thus $\langle\zeta\rangle \leqq k /\left[\alpha_{n}\left(\kappa_{n}\right)+1 / 4\right]$ :

$$
k \frac{B_{3}}{A_{3}} \geqq \frac{2 k}{2\left(\kappa_{n}-x\right)+k /\left[\alpha_{n}(x)+1 / 4\right]} .
$$

This also holds for $k=1 / 2$ with a correction $\left(1-O\left(x^{-1}\right)\right)$ in front of the right-hand side of Eq. (4.21). In fact $\mu(p, q ; x)$ is increasing in $q \in\left[q_{\min }, q_{\max }=\kappa_{n}\right]$, and then putting $q=\kappa_{n}$ yields the lower bound.

The self-consistency condition is obtained be insisting that the lower bound of $k B / A$ is bounded by $\alpha_{\min }+1 / 2$ for $x=\kappa_{n}-\omega=\kappa_{n+1}$, and the most strong condition comes from $k B_{1} / A_{1}$ : for large $k$

$$
\frac{2 k}{2 \omega+(k+1) /\left[\alpha_{\min }+1 / 4\right]} \geqq \alpha_{\min }+\frac{1}{2},
$$

which is equivalent to

$$
2 \omega\left(\alpha_{\min }+\frac{1}{4}\right)+k+1 \leqq 2 k \frac{\alpha_{\min }+1 / 4}{\alpha_{\min }+1 / 2},
$$

from which Eq. (4.4e) follows. Use (4.19c) for small $k$. 
Thus the properties (4.4a-e) are inherited by $g_{n+1}$. As for the convexity of $\alpha_{n+1}$, note that $\alpha_{n}(x)$ is convex and increasing in $x$. Thus $k B_{2} / A_{2}$ is bounded from below by an increasing and convex function. And moreover the lower bounds for other $k B_{i} / A_{i}$ are obviously increasing and convex in $x$. Thus $\alpha_{n+1}(x)$ can be defined to be positive, increasing and convex on $I_{n+1}$.

(case 3) $x \geqq \kappa_{n-\tilde{n}}$ :

Lemma 7. Assume the same conditions in Theorem 4. Then

(1) For fixed $x\left(\geqq \kappa_{n-\tilde{n}}\right)$, the $\left(2-\delta_{k}\right)$ in Theorem 4 can be replaced by $\left[2-\delta_{k}^{(n)}(x)\right]$ that converges to 2 exponentially fast in $n$.

(2) For $x \in\left[O\left(k^{2}\right), \kappa\right]$, let the integer $n_{0}$ be defined by $x \in\left[\kappa-\left(n_{0}+1\right) \omega, \kappa-n_{0} \omega\right]$. Then for $n>n_{0}$ there exists a strictly positive constant $C$ such that

$$
-\left[\ln g_{n}(x)\right]^{\prime} \geqq C 2^{n-n_{0}} .
$$

Proof. (1) We use the fact that $\alpha_{n}(x)$ can be chosen as convex functions: $\alpha_{n}(x+\zeta)+$ $\alpha_{n}(x-\zeta) \geqq 2 \alpha_{n}(x)$ in the present region. Decompose $D$ into $D_{0}, D_{1}$ and $D_{2}$, where

$$
\begin{aligned}
& D_{0}=\{(p, q) \in D ;|p-q| \leqq \sqrt{2} d\}, \\
& D_{2}=\{(p, q) \in D ; 0 \leqq q \leqq \tilde{x}\}, \quad D_{1}=D \backslash\left(D_{0} \cup D_{2}\right),
\end{aligned}
$$

where $\tilde{x}=x-d / \sqrt{2}+d^{2} / 8 x$ and $d$ will be chosen in such a way that $A_{2} /\left(A_{0}+A_{1}\right)$ becomes minimum. Define

$$
\tilde{D}=\left\{(p, q) \in D ; x \leqq q \leqq p,-(\partial / \partial p)\left\{\ln \left[\mu(p, q, x)^{k} f_{n}(p)\right\}<L\right\}\right.
$$

where $\alpha_{n}(x) \gg L \gg 1$. Then

$$
\tilde{D} \subset D_{\varepsilon}=\left\{(p, q) \in D ;(2 \sqrt{x}-\sqrt{q})^{2} \leqq p \leqq(2 \sqrt{x}-\sqrt{q})^{2}+\varepsilon\right\},
$$

where $\varepsilon<$ const. $k\left[\alpha_{n}(x)+1 / 4-L\right]^{-1}$. Then there exists a $d$ such that

$$
A_{1} /\left(A_{0}+A_{2}\right)<\text { const } \alpha_{n}(x)^{-1} \text {. }
$$

See Fig. 2.

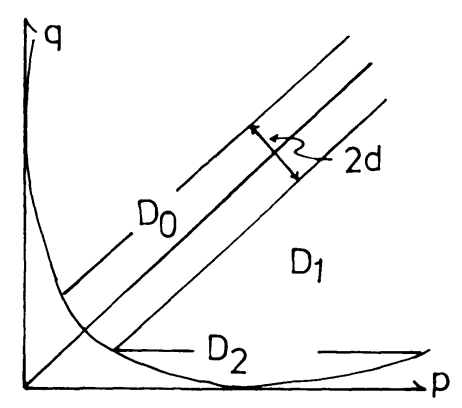

Fig. 2. Large $x$ and large $n$ region in which $-\left[\ln g_{n}(x)\right]^{\prime}$ grows like const $2^{n}$. The rectangle containing the line $p=q$ is thin and may be of order $\alpha_{n}(x)^{-1}$ 
We estimate $k B_{0} / A_{0}$ for this $D_{0}$. Let $p=x+\zeta+\xi, q=x+\zeta-\xi$, where

$$
|\xi| \leqq d / \sqrt{2}, \quad \xi^{2} / 4 x \equiv \zeta_{\min }(\xi)<\zeta<\zeta_{\max }(\xi) \equiv \kappa-x-|\xi| .
$$

Letting $\mu(\zeta, \xi)=\mu(p, q ; x)$, we remark that $d p d q=$ const. $d \zeta d \xi, k \mu(\zeta, \xi)^{k-1}=(\partial / \partial \zeta) \mu(\zeta, \xi)^{k}$ and $\mu(\zeta, \xi)^{k} f_{n}(x+\zeta+\xi) f_{n}(x+\zeta-\xi)$ vanishes at $\zeta=\zeta_{\min }$ and $\zeta=\zeta_{\max }$. Thus by integration by parts, one obtains

$$
\begin{aligned}
k \frac{B_{0}}{A_{0}} & \geqq\left\{1-O\left(x^{-2} \alpha^{-2}\right)\right\} \inf _{(\zeta, \xi)}\left\{-(\partial / \partial \zeta) \ln \left[f_{n}(p) f_{n}(q)\right]\right\} \\
& \geqq\left\{1-O\left(x^{-2} \alpha^{-2}\right)\right\} \inf _{(\zeta, \xi)}\left\{\alpha_{n}(x+\zeta+\xi)+\alpha_{n}(x+\zeta-\xi)+\frac{1}{2}\right\} \\
& \geqq\left\{1-O\left(x^{-2} \alpha^{-2}\right)\right\}\left[2 \alpha_{n}(x)+\frac{1}{2}\right],
\end{aligned}
$$

which together with estimate (4.24) establish Lemma 7 (1).

(2) For $x>O\left(k^{2}\right), n_{0}=[(\kappa-x) / \omega]+O(1)$. Then for $n>n_{0}$,

$$
\alpha_{n+1}(x) \geqq\left[2-\frac{C}{\alpha_{n}(x)}\right]\left[\alpha_{n}(x)+\frac{1}{4}\right]-\frac{1}{2}
$$

$(C=$ constant $)$ which implies that

$$
\alpha_{n+1}(x) \geqq 2^{n-\tilde{n}_{0}}\left(\alpha_{n_{0}}(x)-C^{\prime}\right)+C^{\prime}
$$

with a suitable constant $C^{\prime}$. Q.E.D.

\section{Trajectories of $\left\{g_{n}(x)\right\}$; (II) Transition and High-Temperature Regions}

Iterating the recursion formulas, we reach the region in which $\kappa_{n}$ is no larger than $O\left(k^{2}\right)$ and thus $O(1 / k) \lesssim k / k_{n} \sim k / x$. Thus $\omega=C_{1} k$ depends on $x$ in this region.

For $O(1)<x<O\left(k^{2}\right)$, we may assume $\omega=\omega_{\mathrm{tr}}=O(1)(<1)$ for simplicity at the cost of the speed of the convergence of $\left\{g_{n}\right\}$ to zero. On the other hand $\alpha_{\min }^{\mathrm{tr}} \equiv \alpha_{n}\left(\kappa_{n}\right)$ may be large:

$$
\alpha_{\min }^{\mathrm{tr}}=O(k)+1
$$

Theorem 8. (Transition Region) Let $N_{0}=\left[\left(\kappa-O\left(k^{2}\right)\right) / \omega\right]$ and let $\tilde{\kappa}=\kappa_{N_{0}}=O\left(k^{2}\right)$. Assume $N_{0} \leqq n \leqq N_{0}+O\left(k^{2}\right)$, and let $\kappa_{n}=\kappa_{N_{0}}-\left(n-N_{0}\right) \omega_{\mathrm{tr}}$, where $0<\omega_{\mathrm{tr}}=O(1)$. Then there exist positive monotone increasing and convex functions $\left\{\alpha_{n}(x)\right\}$, where each $\alpha_{n}(x)$ is defined on $I_{n}=\left[\kappa_{n}, \kappa\right]$ and satisfies:

(1) $-\left[\ln g_{n}(x)\right]^{\prime} \geqq \alpha_{n}(x)$,

(2) $\alpha_{n}(x) \geqq\left[2-\delta_{k}\right] \alpha_{n-1}(x)$, for $x \in I_{n-1}$,

(3) $\left[2-\delta_{k}\right] \alpha_{\min } \geqq \alpha_{n}(x) \geqq \alpha_{\min }^{\mathrm{tr}}$, for $x \in\left[\kappa_{n}, \kappa_{n-1}\right]$,

(4) $\alpha_{n}\left(\kappa_{n}\right)=\alpha_{\min }^{\mathrm{tr}}=O(k)+1 \quad(\gg k)$.

Here $\delta_{k}<1, \delta_{k}<$ const $/ k$, and there is a constant $\varepsilon_{k} \ll 1$ such that

$$
2 \alpha_{\min }^{\mathrm{tr}} \omega \leqq k\left(1-\varepsilon_{k}\right) \text {. }
$$

Remark 3. We can refine this theorem as follows: 
(1) For fixed $x(>O(1)), \delta_{k}$ is replaced by $\delta_{k}^{(n)}(x)$ which converges to 0 exponentially fast as $n \rightarrow \infty$.

(2) For fixed $x>O(1)$, there exist positive constants $n_{0}=n_{0}(k, \kappa)$ and $C$ such that $\alpha_{n}(x)>C 2^{n-n_{0}}$.

Proof. Since the proof is essentially equivalent to that of Theorem 4, we sketch the proof. Difficulties lie in the largeness of $(p-q)^{2} / 16 x$ for $x \sim 1$ which is the reason why we set $\alpha_{n}\left(\kappa_{n}\right) \sim O(k)+1$. (i) For $\kappa_{n} \leqq x$, we again decompose $D$ into $D_{1}$ and $D_{2}$, see Fig. 2a. As we have seen already, the contribution $k B_{2} / A_{2}$ is given by (4.11) and then safe and

$$
k \frac{B_{1}}{A_{1}}=\frac{2 k}{\langle\langle\zeta+\xi\rangle},
$$

where $\langle\langle\cdot\rangle\rangle$ is again the expectation value (4.12b). The point is that $\alpha_{\min }^{\mathrm{tr}}=O(k)+1$ $(\gg 1)$ and $\omega_{\mathrm{tr}}$ is small (but the relation $\alpha_{\min }^{\mathrm{tr}} \omega_{\mathrm{tr}}=O(k)$ may be kept.) Namely $f(\zeta)$ decreases rapidly in this region to such an extent that we can use the previous method in the proof of Theorem 4. The remaining discussion is rather clear and therefore omitted.

(ii) $x \in\left[\kappa_{n+1}=\kappa_{n}-\omega^{\mathrm{tr}}\right.$., $\left.\kappa_{n}\right]$. One can apply the proof of Theorem 4 in this case by decomposing $D$ into four regions $D_{0}, \ldots, D_{3}$ as in Sect. 4 (see Fig. 2b). Q.E.D.

Remarks 4. (1) $\omega_{\mathrm{tr}}$ may depend on $x$ sensitively in this region. But we have neglected it and thus the convexity of $\alpha_{n}(x)$ seems to be lost at $x=\kappa_{N_{0}}=O\left(k^{2}\right)$ but is obviously kept for $O(1)<x<\kappa_{N_{0}}$. Therefore there is no difficulty in obtaining the refined version of this theorem which uses the convexity of the function. The reader may choose $\omega_{\mathrm{tr}}$ in such a way that it depends on $x$ smoothly and $\alpha_{n}(x)$ is convex on the whole region.

(2) We have chosen $\alpha_{n}$ so that $\alpha_{n}\left(\kappa_{n}\right)>O(k)+1$. But $\alpha_{n}\left(\kappa_{n}\right)$ increases rapidly in this region and thus there is no essential difference between this and our previous choice $\alpha_{n}\left(\kappa_{n}\right)=O(1)$.

Now let $N_{1}\left(>N_{0}\right)$ be a positive integer such that $\kappa_{n_{1}}=\kappa_{N_{0}}-\left(N_{1}-N_{0}\right) \omega_{\mathrm{tr}}$. For $n>N_{1}, x$ is so small and we have to change our method of the proof. The essential point is that $\kappa_{n}$ decreases to zero exponentially in this region.

Theorem 9. (High Temperature Region.) For $n>N_{1}$, there exist positive increasing and convex functions $\left\{\alpha_{n}(x)\right\}$ and constants $\tau \in[1 / 2,1)$ and $\left[2-\delta_{k}\right] \in(1,2]$. Here $\tau$ can be chosen close to $1 / 2$ and $\delta_{k}<\min \{2 / 3,1 / k\}$ converges to 0 exponentially in $n$ as before. Each $\alpha_{n}(x)$ is defined on $I_{n}=\left[\kappa_{n} \equiv \tau \kappa_{n-1}, \kappa\right]$ and satisfies:

(1) $-\left[\ln g_{n}(x)\right]^{\prime} \geqq \alpha_{n}(x)$.

(2) $2 \alpha_{n-1}(x) \geqq \alpha_{n}(x) \geqq\left[2-\delta_{k}\right] \alpha_{n-1}(x), \quad x \in I_{n-1}$.

(3) $\left[2-\delta_{k}\right] \frac{k}{\kappa_{n}} \geqq \alpha_{n}(x) \geqq \frac{k}{\kappa_{n}}$ if $x \in\left[\kappa_{n}, \kappa_{n-1}\right]$.

(4) $\alpha_{n}\left(\kappa_{n}\right)=\frac{k}{\kappa_{n}}$.

(5) $2 \geqq 2-\delta_{k} \geqq 1 / \tau>1$.

Proof. (i) case of $x \in\left[\kappa_{n}, \kappa_{n-\tilde{n}}=\kappa_{n} \tau^{-\tilde{n}}\right](\tilde{n}=$ small integer): Decompose $D$ into 
$D_{1}$ and $D_{2}$ as before, see Fig. 1a. Then $k B_{2} / A_{2}$ is bounded from below by $2\left[\alpha_{n}(x)+1 / 4\right]-1 / 2 x$. As for $k B_{1} / A_{1 \text { ns }}$ we have:

$$
\frac{1}{2 x}+k \frac{B_{1}}{A_{1}}=\frac{1}{2 x}+k \frac{1-\left\langle\left\langle(\zeta-\xi)^{2}\right\rangle\right\rangle / 16 x^{2}}{\frac{1}{2}\langle\langle\zeta+\xi\rangle\rangle-\left\langle\left\langle(\zeta-\xi)^{2}\right\rangle\right\rangle / 16 x},
$$

where $\langle\langle\cdot\rangle\rangle$ denotes the expectation value $(4.12 \mathrm{~b})$ and $-\left[\ln f_{n}(x+\zeta)\right]^{\prime} \geqq \alpha_{n}(x)+$ $1 / 4>k / \kappa_{n}(\geqq k / x)$. Then by $(4.13 \mathrm{a})\langle\langle\zeta+\xi\rangle\rangle \leqq 2 x$ if $k>1$ and thus

$$
k B_{1} / A_{1} \geqq \frac{2 k}{\langle\langle\zeta+\xi\rangle\rangle} \geqq \frac{2 k}{k+1}\left[\alpha_{n}(x)+\frac{1}{4}\right],
$$

which is larger than $\alpha_{n}(x)+1 / 4$ for $k>1$. For $k=1$ and $1 / 2$, we choose $\tau$ close to 1 and $\left(2-\delta_{k}\right)<2$ close to 2 at the cost of speed of the convergence. From $(5.6 \mathrm{a}-\mathrm{e})$, we have:

$$
\alpha_{n}\left(x \tau^{\ell}\right) \in\left[(\tau(2-\delta))^{\ell} k / \kappa_{n}, \quad(2-\delta)(2 \tau)^{\ell} k / \kappa_{n}\right]
$$

for $x \in\left[\kappa_{n} \equiv C \tau^{n}, \kappa_{n-1}=C \tau^{n-1}\right](C$ is a positive constant). Then if $\tau<1$ is chosen close to 1 and $(2-\delta)<2$ is chosen close to $2, \alpha_{n}(x+\zeta)$ increases rapidly as $\zeta$ increases. This implies that if $x \in\left[\kappa_{n}, \kappa_{n-\tilde{n}}\right]$, then both $k /\langle\zeta\rangle$ and $k /\langle\langle\zeta+\xi\rangle\rangle$ are larger than $2 \alpha_{n}(x)$.

$(k=1)$ Let \langle\rangle$_{0}$ be the expectation value (4.16). Then we have

$$
\langle\langle\zeta+\xi\rangle\rangle=2\langle\zeta\rangle_{0}, \quad\left\langle\left\langle(\zeta-\xi)^{2}\right\rangle\right\rangle=2\left\langle\zeta^{2}\right\rangle_{0}-2\langle\zeta\rangle_{0}^{2} .
$$

Since $\langle\zeta\rangle_{0} \ll \alpha_{n}(x)$ by the choice of $\tau$ and $(2-\delta)$, we have

$$
\frac{B_{1}}{A_{1}} \geqq \frac{1+\left[\langle\zeta\rangle_{0}^{2}-\left\langle\zeta^{2}\right\rangle_{0}\right] / 8 x^{2}}{\langle\zeta\rangle_{0}+\left[\langle\zeta\rangle_{0}^{2}-\left\langle\zeta^{2}\right\rangle_{0}\right] / 8 x} \geqq \frac{1}{\langle\zeta\rangle_{0}} .
$$

The right-hand side can be made larger than $2\left[\alpha_{n}(x)+1 / 4\right]$, and the constraint comes from $k B_{2} / A_{2}+1 / 2 x \geqq 2\left[\alpha_{n}+1 / 4\right]$.

$(k=1 / 2)$ It suffices to consider $B_{1} / 2 A_{1}$ only,

$$
\begin{aligned}
& \frac{1}{2 x}+\frac{\frac{1}{2}\left[1-\left\langle\left\langle(\zeta-\xi)^{2}\right\rangle\right\rangle / 16 x^{2}\right]}{\frac{1}{2}\langle\langle\zeta+\xi\rangle\rangle-\frac{1}{16 x}\left\langle\left\langle(\zeta-\xi)^{2}\right\rangle\right\rangle}, \\
& \left\langle\langle\zeta+\zeta\rangle \leqq \sup _{\theta} \frac{\int d r r^{2}\left[r-\varepsilon_{\theta} r^{2} / 8 x\right]^{-1 / 2} F_{\theta}(r)}{\int d r r\left[r-\varepsilon_{\theta} r^{2} / 8 x\right]^{-1 / 2} F_{\theta}(r)},\right.
\end{aligned}
$$

where $\varepsilon_{0}=[\cos (\theta-\pi / 4) / \cos (\theta+\pi / 4)]^{2}$. We again choose $\tau<1$ close to $1,2-\delta$ close to 2 . Then this again can be made larger than $2\left[\alpha_{n}+1 / 4\right]$.

(ii) case of $x \in\left[\kappa_{n+1}=\tau \kappa_{n}, \kappa_{n}\right]$ : Decompose $D$ into four pieces $D_{0}, \ldots, D_{3}$ as before. Here $k B_{0} / A_{0} \geqq k /\left(\kappa_{n}-x\right)$ and $k B_{2} / A_{2}$ obeys the bound in Lemma $6(1)$. Moreover

$$
\begin{aligned}
k \frac{B_{1}}{A_{1}} & =\frac{2 k\left[1-\left\langle\left\langle\varepsilon_{1}\right\rangle\right\rangle / 16 x^{2}\right]}{2 \Delta+\langle\langle\zeta+\xi\rangle\rangle-\left\langle\left\langle\varepsilon_{1}\right\rangle\right\rangle / 16 x}, \\
k \frac{B_{3}}{A_{3}} & =\frac{2 k\left[1-\left\langle\varepsilon_{3}\right\rangle / 16 x^{2}\right]}{2 \Delta+\langle\zeta\rangle-\left\langle\varepsilon_{3}\right\rangle / 16 x},
\end{aligned}
$$


where $\Delta=\kappa_{n}-x, \varepsilon_{1}=(\zeta-\xi)^{2}, \varepsilon_{3}=\zeta^{2}$. If

$$
\begin{aligned}
\Delta+\frac{1}{2}\langle\langle\zeta+\xi\rangle\rangle & \leqq x, \\
\Delta+\frac{1}{2}\langle\zeta\rangle & \leqq x,
\end{aligned}
$$

then we can omit $\left\langle\left\langle\varepsilon_{1}\right\rangle\right\rangle$ and $\left\langle\varepsilon_{3}\right\rangle$ to obtain the lower bounds and we find:

$$
\begin{aligned}
& k \frac{B_{1}}{A_{1}} \geqq \frac{2 k}{2 \Delta+\langle\langle\zeta+\xi\rangle\rangle} \geqq \frac{2 k}{2 \Delta+(k+1) /\left[\alpha_{n}\left(\kappa_{n}\right)+1 / 4\right]}, \\
& k \frac{B_{3}}{A_{3}} \geqq \frac{2 k}{2 \Delta+\langle\zeta\rangle} \geqq \frac{2 k}{2 \Delta+k /\left[\alpha_{n}\left(\kappa_{n}\right)+1 / 4\right]} .
\end{aligned}
$$

If $k>1$, we have

$$
\Delta+\langle\langle\zeta+\xi\rangle\rangle\left\langle\left(\kappa_{n}-x\right)+\frac{k+1}{2 k} \kappa_{n}\right.
$$

This is less than $x$ if $x>[(3 k+1) / 4 k] \kappa_{n}$ : there exists $\tau \in[1 / 2,1)$ such that our assertion holds if $k>1$. We now show that $\tau$ approaches $1 / 2$ as $k$ tends to infinity. By applying Lemma 5 and the lemmas in the appendix, we see that for $k>1$,

$$
\begin{aligned}
\Delta+\frac{1}{2}\langle\zeta\rangle & \leqq \Delta+\frac{1}{2}\left[\frac{k-1}{\alpha_{n}\left(\kappa_{n}\right)+1 / 4}-2 \Delta\right]+\frac{\sqrt{k}}{\alpha_{n}\left(\kappa_{n}\right)}+c_{1}(k) \\
& =\frac{1}{2}\left[\frac{k-1}{\alpha_{n}\left(\kappa_{n}\right)+1 / 4}\right]+\frac{\sqrt{k}}{\alpha_{n}\left(\kappa_{n}\right)}+c_{1}(k), \\
\Delta+\frac{1}{2}\langle\langle\zeta+\xi\rangle\rangle & \leqq \Delta+\frac{1}{2}\left[\frac{k-1}{\alpha_{n}\left(\kappa_{n}\right)+1 / 4}-2 \Delta\right]+\frac{\sqrt{k}}{\alpha_{n}\left(\kappa_{n}\right)}+c_{2}(k) \\
& =\frac{1}{2}\left[\frac{k-1}{\alpha_{n}\left(\kappa_{n}\right)+1 / 4}\right]+\frac{\sqrt{k}}{\alpha_{n}\left(\kappa_{n}\right)}+c_{1}(k),
\end{aligned}
$$

where $c_{i}(k)$ are of order $o\left(\kappa_{n} / \sqrt{k}\right)$. Thus if $k$ is large, then both $\Delta+\langle\zeta\rangle / 2$ and $\Delta+\langle\langle\zeta+\xi\rangle\rangle / 2$ are close to $\kappa_{n} / 2$, which means that $\tau$ can be taken as small as $1 / 2$. This is consistent with the standard theory of large deviations in probability theory. Remark that the right-hand-sides of Eqs. $(5.11 \mathrm{a}, \mathrm{b})$ and $(5.13 \mathrm{a}, \mathrm{b})$ are all increasing and convex functions of $x$.

We again improve the bounds for small $k(1,1 / 2)$ by choosing $\tau$ close to 1 and $2-\delta$ close to 2 .

$(k=1)$ Let $\langle\cdot\rangle_{0}$ be the expectation value (4.16). Then we have:

$$
\frac{B_{1}}{A_{1}} \geqq \begin{cases}\frac{1}{\kappa_{n}-x+\langle\zeta\rangle_{0}} & \text { if } x>\kappa_{n}-x+\langle\zeta\rangle_{0} \\ \frac{1+\left[\langle\zeta\rangle_{0}^{2}-\left\langle\zeta^{2}\right\rangle_{0}\right] / 8 x^{2}}{\kappa_{n}-x+\langle\zeta\rangle_{0}+\left[\langle\zeta\rangle_{0}^{2}-\left\langle\zeta^{2}\right\rangle_{0}\right] / 8 x} & \text { otherwise }\end{cases}
$$

Note that since $\tau$ is close to 1 , then $0<\kappa_{n}-x<C \tau^{n}(1-\tau) \ll \kappa_{n+1}$ and moreover $\langle\zeta\rangle_{0}$ is smaller than $1 / \alpha_{n}\left(\kappa_{n}\right)$. Then

$$
\frac{1}{2 x}+\frac{B_{1}}{A_{1}} \geqq \frac{1}{2 x}+\frac{1}{\kappa_{n}-x+\langle\zeta\rangle_{0}} \gg \frac{1}{2 x}+\frac{1}{\kappa_{n+1}} .
$$


The analysis of $B_{3} / A_{3}$ is similar and gives the lower bound larger than that of $B_{1} / A_{1}$. Note that $\alpha$ is chosen increasing and convex on $\left[\kappa_{n+1}, \kappa_{n}\right]$.

$(k=1 / 2)$ It suffices to consider $B_{1} / 2 A_{1}$ only. We consider

$$
\frac{1}{2 x}+\frac{\frac{1}{2}\left[1-\left\langle\left\langle(\zeta-\xi)^{2}\right\rangle\right\rangle / 16 x^{2}\right]}{\kappa_{n}-x+\frac{1}{2}\langle\langle\zeta+\xi\rangle\rangle-\frac{1}{16 x}\left\langle\left\langle(\zeta-\xi)^{2}\right\rangle\right\rangle},
$$

where $x>\kappa_{n} / 2$ and

$$
\left\langle\zeta+\xi 》 \leqq \sup _{\theta} \frac{\int d r r^{2}\left[2 \Delta+r-r^{2} / 8 x\right]^{-1 / 2} F_{\theta}(r)}{\int d r r\left[2 \Delta+r-r^{2} / 8 x\right]^{-1 / 2} F_{\theta}(r)}\right.
$$

with $\Delta=\kappa_{n}-x$. Again by choosing $\tau$ close to 1 and $(2-\delta)$ close to 2 , we can make $\langle\langle\zeta+\xi\rangle\rangle$ very small.

(iii) case of $x>\kappa_{n-\tilde{n}}$ : Note that $\left\{\alpha_{n}(x)\right\}$ are chosen convex and increasing. Then we apply Lemma 7. Q.E.D.

Since the Hamiltonian part becomes negligible $\left(O\left(\tau^{n}\right)\right)$ in the high-temperature region, one expects that $\tau$ can be chosen as small as $1 / 2$ (see $[10,11]$ ). But the present estimate using a priori bound of two-ball-overlap is weak for this. Refined inductive estimates may enable us to prove this conjecture.

Remarks 5. (1) The values of $2-\delta_{k}$ and $\tau$ are far from the optimal values 2 and $1 / 2$ respectively for small $k$. For this, we estimate $A_{1} / A_{2}$ which turns out to be $O(1)$. Then we can know to what extent $B_{2} / A_{2}$ contributes to $B / A$, see (4.8).

(2) From these discussions, it is now clear that we can extend $g_{0}$ to functions which decrease faster than any Gaussian functions for large $x$.

\section{Correlation Functions}

Without loss of generalities, we set $L=\sqrt{2}$ and consider the two-point correlation functions only. Let $x, y \in Z^{2}$ and consider sufficiently large hierarchical lattices containing $x$ and $y$. Let $\langle\cdot\rangle$ be the expectation values with respect to the probability measure of the present system. We have the decompositions

$$
\begin{aligned}
\vec{\phi}(x) & =(C \vec{\phi})\left(x_{1}\right) \pm \vec{z}\left(x_{1}\right), \\
(C \vec{\phi})\left(x_{1}\right) & =\left(C^{2} \phi\right)\left(x_{2}\right) \pm z\left(x_{2}\right), \ldots .
\end{aligned}
$$

Here for $x \in[2 i, 2 i+2) \times\{j\}$ we set $x_{1}=(i, j) \in Z^{2}$, and similarly for $x_{1} \in\{i\} \times$ $[2 j, 2 j+2)$ we set $x_{2}=(i, j) \in Z^{2}$ and so on, $C$ the block spin operator and $z$ 's are the fluctuation field. Thus in the present system of the hierarchical structure,

$$
\lim _{\Lambda \uparrow Z^{2}}\langle\vec{\phi}(x) \vec{\phi}(y)\rangle_{\Lambda}^{h c L}= \pm H_{m}+\sum_{m+1}^{\infty} H_{n},
$$

where

$$
\begin{gathered}
H_{n}=\lim _{\ell \rightarrow \infty} H_{n}^{(\ell)}, \\
H_{n}^{(\ell)}=\int h_{n}^{(\ell)}\left(\phi^{2}\right) d \vec{\phi}, \\
h_{n}^{(\ell+1)}\left(\vec{\phi}^{2}\right)=\frac{\int g_{n+\ell}\left((\vec{\phi}+\vec{z})^{2}\right) h_{n}^{(\ell)}\left((\vec{\phi}-\vec{z})^{2}\right) e^{-\vec{z}^{2} / 2} d \vec{z}}{\int g_{n+\ell}\left((\vec{\phi}+\vec{z})^{2}\right) g_{n+\ell}\left((\vec{\phi}-\vec{z})^{2}\right) e^{-\vec{z}^{2} / 2} d \vec{z} d \vec{\phi}}
\end{gathered}
$$


for $\ell=0,1, \ldots$ and especially

$$
h_{n}^{(0)}=h_{n}^{(0)}\left((\vec{\phi}-\vec{z})^{2}, \vec{z}^{2}\right)=g_{n}\left((\vec{\phi}-\vec{z})^{2}\right) \vec{z}^{2} .
$$

$m$ is the smallest integer such that $x_{m}=y_{m}$. We set dist $(x, y)=2^{m / 2}$. and we normalize $\left\{g_{n}\left(\phi^{2}\right)\right\}$ so that $\int g_{n}\left(\phi^{2}\right) d \vec{\phi}=1$.

Theorem 10. For $\ell \geqq 1$ and for large $n$ such that $\kappa_{n} \ll 1$,

$$
H_{n}^{(\ell)}<C(\kappa) 2^{-n}
$$

uniformly in $\ell$, and thus

$$
H_{n}<C(\kappa) 2^{-n}
$$

Proof. We first prove that

$$
\int \vec{\phi}^{2} g_{n}\left(\vec{\phi}^{2}\right) d \vec{\phi}<C_{1}(\kappa) 2^{-n}
$$

uniformly in $n$. In fact

$$
\begin{aligned}
\text { L.H.S. } & =\frac{\int g_{n-1}\left(\phi_{1}^{2}\right) g_{n-1}\left(\phi_{2}^{2}\right) \exp \left[-\frac{1}{8}\left(\vec{\phi}_{1}-\vec{\phi}_{2}\right)^{2}\right]\left(\vec{\phi}_{1}+\vec{\phi}_{2}\right)^{2} d \vec{\phi}_{1} d \vec{\phi}_{2}}{4 \int g_{n-1}\left(\phi_{1}^{2}\right) g_{n-1}\left(\phi_{2}^{2}\right) \exp \left[-\frac{1}{8}\left(\vec{\phi}_{1}-\vec{\phi}_{2}\right)^{2}\right] d \vec{\phi}_{1} d \vec{\phi}_{2}} \\
& \leqq \frac{\int g_{n-1}\left(\phi_{1}^{2}\right) g_{n-1}\left(\phi_{2}^{2}\right)\left(\vec{\phi}_{1}+\vec{\phi}_{2}\right)^{2} d \vec{\phi}_{1} d \vec{\phi}_{2}}{4 \int g_{n-1}\left(\phi_{1}^{2}\right) g_{n-1}\left(\phi_{2}\right)\left[1-\varepsilon\left(\vec{\phi}_{1}, \vec{\phi}_{2}\right)^{2}\right] d \phi_{1} d \phi_{2}},
\end{aligned}
$$

where $\varepsilon\left(\phi_{1}, \phi_{2}\right)=1-\exp \left[-\left(\phi_{1}-\phi_{2}\right)^{2} / 8\right]<\min \left\{\left(\phi_{1}^{2}+\phi_{2}^{2}\right) / 4,1\right\}$. Thus using $\int\left(\vec{\phi}_{1} \vec{\phi}_{2}\right) g_{n-1}\left(\phi_{1}^{2}\right) d \vec{\phi}_{1}=0$ and our previous estimates, we have

$$
\int \vec{\phi}^{2} g_{n}\left(\vec{\phi}^{2}\right) d \phi<\left[1+C_{2} \tau^{n-1}\right] \frac{1}{2} \int \vec{\phi}^{2} g_{n-1}\left(\vec{\phi}^{2}\right) d \phi,
$$

and thus we next use indication.

We turn to $H_{n}^{(\ell)}=\int h_{n}^{(\ell)}(\vec{\phi}) d \vec{\phi}$. Integrate the right-hand side of Eq. (6.6) over $\vec{\phi}$. Repeating the above arguments, we have

$$
H_{n}^{(\ell)} \leqq\left[1+C_{2} \tau^{n+\ell-1}\right] H_{n}^{(\ell-1)},
$$

and thus by induction we finally have

$$
\leqq \prod_{i=1}^{\ell-1}\left[1+C_{2} \tau^{n+i}\right] H_{n}^{(0)}
$$

where $H_{n}^{(0)}$ is the quantity obtained by replacing $\left(\vec{\phi}_{1}+\vec{\phi}_{2}\right)^{2}$ in the numerator of Eq. (6.9) by $\left(\vec{\phi}_{1}-\vec{\phi}_{2}\right)^{2} / 4$. Then the previous arguments directly show that $H_{0}^{(n)}<$ const $2^{-n}$. Q.E.D.

Therefore the two point correlation function is given by

$$
\begin{aligned}
\langle\vec{\phi}(x) \vec{\phi}(y)\rangle^{h c l} & = \pm H_{m}+\sum_{m+1} H_{n} \\
& =O(1) 2^{-m}=O(1) d(x, y)^{-2} .
\end{aligned}
$$

This is a pathology due to the hierarchical structure, and this decay rate is saturated by the hierarchical massive gaussian model [Remark 1(1)].

This may be seen from another point of view: Consider an averaged spin $\vec{\Phi}=|\Lambda|^{-1}\left(\sum \vec{\phi}_{i}\right)$. Then $\left\langle\vec{\Phi}^{2}\right\rangle^{h c l}\left\langle\right.$ const $|\Lambda|^{-1}$. Then if $\langle\vec{\phi}(x) \vec{\phi}(y)\rangle$ is monotone decreasing in $|x-y|$ (this is not satisfied in the hierarchical system but is satisfied 
by the usual Heisenberg or by sigma model), this roughly means $\langle\phi(\vec{x}) \phi(\vec{y})\rangle \lesssim$ $\left(|x-y|^{2}+1\right)^{-1}$. The Aizenman-Simon inequality [12] means exponential clusterings. What is important to get this main result is that $g_{n}\left(\phi^{2}\right)$ converges to zero exponentially fast in $n$, like $\tau^{n}$, no matter how $\tau$ is close to 1 .

\section{Small $N$ Models $(N=2,3)$}

For $N=2,3$, we have Theorem 2 and thus have the same results:

Theorem 11. For $N=2$ or for $N=3$, the flow $\left\{g_{n}\right\}$ is attracted into the high temperature region by the renormalization group recursion formulas of $D y$ son-Wilson type for any $g_{0}\left(\operatorname{supp} g_{0} \subset[0, \kappa]\right)$. The two-point correlation functions decay like $[d(x, y)]^{-2}$.

We sketch the proof which is almost equivalent to the previous one except that we have to integrate over $D=\left\{\left(p_{1}, \ldots, p_{4}\right) \in[0, \kappa]^{4} ; \min \left|\sum \pm \sqrt{p_{i}}\right| \leqq 4 \sqrt{x} \leqq \sum \sqrt{p_{i}}\right\}$ which is convex, see (3.8), (3.12). Note that we also have the integration by parts formula:

$$
\begin{aligned}
& -\frac{\partial}{\partial x}\left[\left(\sum \sqrt{p}_{i} / 4\right)^{2}-x\right]^{\tilde{k}} \\
& \quad=4 \frac{\partial}{\partial p_{1}}\left[\left(\sum{\sqrt{p_{i}}}_{i} / 4\right)^{2}-x\right]^{\tilde{k}}+\sum_{j \neq 1} \frac{1}{4}\left(1-\sqrt{p_{j} / p_{1}}\right) \tilde{k}\left[\left(\sum{\sqrt{p_{i}}}_{i} / 4\right)^{2}-x\right]^{\tilde{k}-1} .
\end{aligned}
$$

Assume the large field region and let $x \geqq \kappa_{n}$. Then we decompose $D$ into several pieces including $D_{1}=\left\{p_{i} \geqq x ; i=1 \sim 4\right\}$. Introduce the polar coordinate and choose $\omega$ small to make $B_{1} / A_{1}$ large. For other regions, use the integration by parts to get the factor 4. This is the method used for $N=3,4$. For $x \in\left[\kappa_{n+1}=\kappa_{n}-\omega, \kappa_{n}\right]$, decompose $D$ into several pieces including $D_{0}=\left\{p_{i}<\kappa_{n}: i=1 \sim 4\right\}$. Then $k B_{0} / A_{0}$ is bounded by the a priori bound. Thus our previous analysis implies that the flow $\left\{g_{n}\right\}$ is driven into the high temperature region.

To extend Lemma 7, we first define $D_{0}=\left\{\left\{p_{i}\right\} \in D ;\left|p_{i}-p_{j}\right| \leqq \sqrt{2} d\right\}$ and next $D_{2} \subset D \backslash D_{0}$, region separated by hyperplanes which contain $\partial D \cap \partial D_{0}$ and are parallel to the coordinate planes. The contributions from $D_{0}$ and $D_{2}$ are estimated by the integration by parts which yields factor 4 . We choose $d$ so that the contribution from $D \backslash\left(D_{0} \cup D_{2}\right)$ becomes minimum.

In the small field region, since the hierarchical Hamiltonian is small, the system is essentially a sum of independent spins. The $\left\{g_{n}(x)\right\}$ are controlled by choosing $\tau$ close to 1 and $(4-\delta)$ close to 4 , as was done for the $N=4,5$ models. Thus $g_{n}(x)$ converges to zero like const $\tau^{n}$ in this region and then the two-point correlation functions decay like $[d(x, y)]^{-2}$. The point to get this result is that the boundary effect is bounded by $O(1)$ uniformly in $\Lambda$.

\section{Conclusions and Discussions}

We have shown that the two-dimensional $O(N)$ vector (Heisenberg) model with hierarchical interactions is driven into the high temperature region by the 
Wilson-Kadanoff block spin transformations if $N \geqq 2$, and thus the (truncated) correlation function decay with the velocity of the massive free model.

Therefore the present approximate formula fails to exhibit the KosterlitzThouless transition (for $N=2$ at least). This is because the boundary effects, which works ferromagnetically are bounded by $O(1)$ (with unit of $\phi^{2}$ ) for all distance scales and magnitudes of block spins in the present system. Thus the probabilistic force wins through all the distance scales.

In [7], Bleher and Major discussed a similar model ( $d$-dimensional hierarchical model with $d>2$ ) in which the strength of the effective Hamiltonian is as strong as $O\left(|\partial \Lambda|^{\varepsilon}\right)=O\left(2^{\varepsilon n / 2}\right)$. Namely they consider the model in which the correction term $\exp \left[\left(L^{2} \varphi-\sum \phi^{2}(x)\right) / 4\right]$ is replaced by $\exp \left[c^{n}\left(L^{2} \varphi^{2}-\sum \phi^{2}(x)\right) / 4\right]$, where $c=2^{1-2 / d}$ and $L=2^{1 / d}$. They showed that the system exhibits the spontaneous magnetization whenever $d>2$, if the parameter $\kappa$ (inverse temperature) is sufficiently large.

So what about our results for $N \geqq 3$ ? To what extent is asymptotic freedom stronger than the boundary effects? What we have shown is that the perturbative asymptotic freedom is purely geometric and even the $N=2$ model has this property. Therefore the difference between the abelian one and non-abelian ones is, even if it exists, very subtle. Therefore our conventional wisdom is not yet established and remains to be investigated from several possible points of view [13-17].

\section{Appendix}

Lemma A.1. Let $f(x)$ and $g(x)$ be positive functions such that $-[\ln g(x)]^{\prime}>$ $-[\ln f(x)]^{\prime}$ and $\operatorname{supp} f=\operatorname{supp} g=[0, \kappa]$. Define

$$
\langle\cdot\rangle(f ; a, b, c ; \lambda)=\frac{\int_{0}^{\lambda}\left(a+b x-c x^{2}\right)^{k}(\cdot) f(x) d x}{\int_{0}^{\lambda}\left(a+b x-c x^{2}\right)^{k} f(x) d x},
$$

where $k \geqq 0, a, b, c \geqq 0$ and $a+b x-c x^{2}>0$ for $0 \leqq x \leqq \lambda$. Then for $m \geqq 0,\left\langle x^{m}\right\rangle$ $(f ; a, b, c ; \lambda)$ is monotone decreasing in $c$, and is increasing in $b$ and $\lambda$. If $c=0$, then $\left\langle x^{m}\right\rangle(f ; a, b, 0 ; \lambda)$ is monotone decreasing in a. Moreover

$$
\left\langle x^{m}\right\rangle(f ; a, b, c ; \lambda) \geqq\left\langle x^{m}\right\rangle(g ; a, b, c ; \lambda) .
$$

Proof. Set $\mu(x)=a+b x-c x^{2},\langle x\rangle_{f}=\langle x\rangle(f ; a, b, c ; \lambda)$ and etc. For $m=1$,

$$
\frac{d}{d b}\langle x\rangle_{f}=C_{1} k \int d x d y f(x) f(y) \mu(x)^{k-1} \mu(y)^{k-1}\left[a(x-y)^{2}+\operatorname{cxy}(x-y)^{2}\right]
$$

by using the symmetry, where $C_{1}>0$. Same for $m>1$. Similarly

$$
\begin{aligned}
\frac{d}{d c}\langle x\rangle_{f}= & C_{2} k \int d x d y f(x) f(y) \mu(x)^{k-1} \mu(y)^{k-1} \\
& \cdot\left[-a(x+y)(x-y)^{2}-b x y(x-y)^{2}\right]<0,
\end{aligned}
$$


where $C_{2}>0$. Moreover

$$
\frac{d}{d \lambda}\langle x\rangle_{f}=C_{3} \mu(\lambda) \lambda f(\lambda) \int_{0}^{\lambda} \mu(x)^{k}(1-x / \lambda) f(x) d x \geqq 0 .
$$

It is obvious that $\langle x\rangle_{f}$ is decreasing in a if $c=0$. Finally

$$
\langle x\rangle_{f}-\langle x\rangle_{g}=C_{4} \int d x d y \mu(x)^{k} \mu(y)^{k}(x-y)[f(x) g(y)-f(y) g(x)],
$$

where $C_{4}>0$ and $[\ldots] \geqq 0$ if $x \geqq y$. In fact

$$
[\ldots]=f(y) g(x)\left[\exp \left(\int_{y}^{x}[\ln f(t)-\ln g(t)]^{\prime} d t\right)-1\right]
$$

and then the integrand is positive. Q.E.D.

This lemma applies also for $f(\zeta) \equiv \zeta^{\ell} f(\zeta)$ and $g(\xi)=\xi^{\ell} g(\xi)$ since $f$ and $g$ again satisfy $[f(x) g(y)-f(x) g(y)](x-y) \geqq 0$.

Lemma A.2. In Lemma A.1, set $a=1, b=\alpha / k$ and $c=0$. Assume that $-[\ln f(x)]^{\prime} \geqq$ $\alpha>0$. Then

$$
\begin{gathered}
\langle x\rangle(f ; 1, \alpha / k, 0 ; \lambda) \leqq\left(\frac{\sqrt{2 k}}{\sqrt{\pi}}+c_{1}(k)\right) \frac{1}{\alpha}, \\
\frac{\left\langle x^{2}\right\rangle(f ; 1, \alpha / k, 0 ; \lambda)}{\langle x\rangle(f ; 1, \alpha / k, 0 ; \lambda)} \leqq\left(\sqrt{\pi k}+c_{2}(k)\right) \frac{1}{\alpha},
\end{gathered}
$$

where $c_{i}(k)$ is a constant less than 1 for all $k \geqq 1 / 2$.

Proof. We may assume $\lambda=\infty$. Introduce a new variable $\zeta=\alpha x$ and let

$$
\begin{gathered}
\left(1+\frac{\zeta}{k}\right)^{k}=\exp \left[\zeta-\frac{\zeta^{2}}{2 k}+k \delta(\zeta / k)\right], \\
\delta(\zeta / k)=\int_{0}^{\zeta / k} \frac{s^{2}}{1+s} d s<\frac{1}{3}(\zeta / k)^{3} .
\end{gathered}
$$

The lower bound is attained when $f(x)=e^{-\alpha x / k}$. We need to estimate

$$
\begin{aligned}
& \int_{0}^{\infty} \exp \left[-\left(\zeta^{2} / 2 k\right)\right](\cdot) d \zeta+\int_{0}^{\infty} \exp \left[-\left(\zeta^{2} / 2 k\right)\right]\left[e^{k \delta}-1\right](\cdot) d \zeta \\
& =\int_{0}^{\infty} \exp \left[-\left(\zeta^{2} / 2 k\right)\right](\cdot) d \zeta+\int_{0}^{1} d t \int_{0}^{\infty} \exp \left[-\left(\zeta^{2} / 2 k\right)+t k \delta(\zeta / k)\right] k \delta(\zeta / k)(\cdot) d \zeta
\end{aligned}
$$

where the first integral is gaussian and the second one is bounded by $\int_{0}^{\infty} \exp \left[-\zeta^{2} / 2 k+k \delta(\zeta / k)\right] k \delta(\zeta / k)(\cdot) d \zeta$. This integral is estimated by decomposing $[0, \infty)$ into $I_{1}=[0, k)$ and $I_{2}=[k, \infty)$. On $I_{1} \exp \left[-\zeta^{2} / 2 k+k \delta(\zeta / k)\right] \sim \exp \left[-\zeta^{2} / 2 k+\right.$ $\left.\xi^{3} / 3 k^{2}\right]$ and on $I_{2} \exp \left[-\zeta^{2} / 2 k+k \delta(\zeta / k)\right] \sim 2^{k} e^{-\zeta}=(2 / e)^{k} e^{-(\zeta-k)}$. Thus $c_{i}(k)$ are bounded by a constant uniformly in $k$ and tend to zero as $k \rightarrow \infty$. Easy calculations show that $c_{i}(k)$ are $<1$. Q.E.D.

Lemma A.3. In Lemma A.1. set $\lambda=\infty, b=1$ and $c=0$. Assume that $-[\ln f(\zeta)]^{\prime} \geqq$ $\alpha>0$ and $\zeta_{0} \equiv \frac{k}{\alpha}-a \geqq 0$. Then 


$$
\langle\zeta\rangle=\zeta_{0}+\delta \zeta_{1}, \quad\left\langle\zeta^{2}\right\rangle /\langle\zeta\rangle=\zeta_{0}+\delta \zeta_{2},
$$

where $\delta \zeta_{1}$ (respectively $\delta \zeta_{2}$ ) is bounded from above by the right-hand side of Eq. $(A .1)$ (respectively the right-hand side of Eq. (A.2)).

Proof. $\exp [k \ln (a+\zeta)-\alpha \zeta]=\exp \left[k \ln \left(\frac{k}{\alpha}+\left(\zeta-\zeta_{0}\right)\right)-\alpha \zeta\right]$. Expand the righthand side and use Lemma A.2. Q.E.D.

Acknowledgements. This paper was completed through discussions with T. Miwa, T. Hara, T. Hattori, K. Kondo, K. Gawedzki. Part of this work was done while the author was visiting the Kings College of London and ETH. I would like to thank R. Streater and J. Fröhlich for their hospitalities extended at these institutes.

\section{References}

1. Wilson, K. G.: Confinement of quarks. Phys. Rev. D10, 2445 (1974)

2. Wilson, K. G.: Renormalization group and critical phenomena. Rev. Mod. Phys. 55, 583(1983) and references cited therein

3. Polyakov, A. M.: Interactions of Goldstone Bosons and restoration of symmetries in two dimensions. Phys. Lett. 59B, 79 (1975)

4. Wilson, K. G. and Kogut. K.: Renormalization group and $\varepsilon$-expansion. Phys. Rep. 12C (1974)

5. Ma, S. K.: Modern theory of critical phenomena, Chap. 8. Reading, MA.: Benjamin 1976

6. Gawedzki, K., Kupiainen, A.: Non-Gaussian fixed points of the block spin transformation, Hierarchical model approximation. Commun. Math. Phys. 89, 191 (1983): Asymptotic freedom beyond perturbation theory, in Les Houches 1984, Critical Phenomena, random systems, gauge theories Part 1. Course 4, Amsterdam-New York: North Holland 1984 Osterwalder, K, Stora, R. (eds.)

7. Bleher, P. M., Major, P.: The Large scale limit of Dyson's hierarchical vector valued model of low temperature, Commun. Math. Phys. 125, 43 (1989); The large scale limit of Dyson's hierarchical vector valued model of low temperature, the non-Gaussian case. Ann. l'Inst. Henri Poincaré, Theor. Phys., 49, 7 (1988)

8. Ito, K. R.: Origin of asymptotic freedom in non-Abelian field theories. Phys. Rev. Lett. 58, 439 (1987)

9. Ito, K. R.: Renormalization group on hierarchical lattices and beyond, Prog. Theor. Phys. [Suppl.] No. 92, 46 (1987)

10. Petrov, V. V.: Limit theorem for large deviations violating Cramer's condition I, II. Am. Math. Transl. (S)7, 235-253, 254-280 (1967) Am. Math. Soc.

11. Richter, W.: A more precise form of inequality of B. N. Bernstein for large deviation. Am. Math. Soc. Transl. (S)4, 225-231, (1965), Am. Math. Soc.

12. Simon, B., Aizenman, M.: Ward Identities and Decay of Correlations. Commun. Math. Phys. 77, 137 (1980)

13. Seiler, E., Stamatescu, I. O., Linke, V., Patrasciou, A.: Critical behavior, scaling and universality in some two-dimensional spin models. Nucl. Phys. B305 [FS23] 623 (1988)

14. Patrasciou, A.: Mass gap in non-abelian sigma models and gauge theories. Phys. Rev. Lett. 58, 2285 (1987)

15. Ito, K. R.: Kosterlitz-Thouless Type Transitions in Two-Dimensional Non-Abelian field theories. Phys. Rev. Lett. 64, 2839 (1990)

16. Heller, U. M.: Monte-Carlo renormalization group investigation of the two-dimensional $O(4)$ sigma model. Phys. Rev. Lett. 60, 2235 (1988)

17. Wolff, U.: Continuum behaviors in the lattice $O(3)$ non-linear sigma model. Nucl. Phys. B334, 581 (1990) 